\title{
Simultaneous optimization of donor and acceptor substrate specificity for transketolase by a small but smart library
}

\author{
Haoran $\mathrm{Yu}^{\mathrm{a}}$, Roberto Icken Hernández López ${ }^{\mathrm{a}}$, David Steadman ${ }^{\mathrm{b}}$, Daniel Méndez-Sánchez ${ }^{\mathrm{b}}$, \\ Sally Higson ${ }^{b}$, Armando Cázares-Körner ${ }^{b}$, John M. Wardª, Helen C. Hailes ${ }^{b}$, Paul A. Dalby ${ }^{a *}$ \\ a Department of Biochemical Engineering, University College London, Gordon Street, \\ London, WC1H OAH, United Kingdom \\ ${ }^{b}$ Department of Chemistry, University College London, 20 Gordon Street, London WC1H \\ OAJ, United Kingdom
}

*Correspondence and requests for materials should be addressed to Paul A. Dalby. Department of Biochemical Engineering, University College London, Gordon Street, London, WC1H 0AH, United Kingdom. Email: p.dalby@ucl.ac.uk; Tel: +44 (0)20 76799566

Running title: Engineer substrate specificity of transketolase

\begin{abstract}
Abbreviations: DXS, 1-deoxy-D-xylulose-5-phosphate synthase; FBA, formylbenzoic acid; HPA, hydroxypyruvate; NMR, nuclear magnetic resonance spectroscopy; PAC, phenylacetylcarbinol; ThDP, thiamine diphosphate; TK, transketolase; TKgst, TK from Geobacillus stearothermophilus; WT, wild type
\end{abstract}

Keywords: protein engineering, transketolase, two substrates specificity, small but smart library, docking

Conflicts of Interest: None 


\begin{abstract}
A narrow substrate range is a major limitation in exploiting enzymes more widely as catalysts in synthetic organic chemistry. For enzymes using two substrates, the simultaneous optimization of both substrate specificities, is also required for the rapid expansion of accepted substrates. Transketolase catalyses the reversible transfer of a $\mathrm{C}_{2^{-}}$ ketol unit from a donor substrate to an aldehyde acceptor and suffers the limitation of narrow substrate scope for widely industrial applications. Herein, transketolase from $E$. coli was engineered to simultaneously accept both pyruvate as a novel donor substrate, and unnatural acceptor aldehydes, including propanal, pentanal, hexanal and 3-formylbenzoic acid. Twenty single-mutant variants were firstly designed and characterized experimentally. Beneficial mutations were then recombined to construct a small but smart library. Screening of this library identified the best variant with a 9.2-fold improvement in the yield towards pyruvate and propionaldehyde, relative to WT. Pentanal and hexanal were used as acceptors to determine stereoselectivities of the reactions, which were found to be higher than $98 \%$ ee for the $S$ configuration. Three variants were identified to be active for the reaction between pyruvate and 3-formylbenzoic acid. The best variant was able to convert $47 \%$ of substrate into product within $24 \mathrm{~h}$, whereas no conversion was observed for WT. Docking experiments suggested a cooperation between the mutations responsible for donor and acceptor acceptances, that would promote the activity towards both the acceptor and donor. The variants obtained have the potential to be used for developing catalytic pathways to a diverse range of high-value products.
\end{abstract}




\section{Introduction}

The limited degree of substrate acceptance has been a major limitation in exploiting enzymes as catalysts in synthetic organic chemistry [1]. The fourth wave of biocatalysis is approaching [2] and the ability to discover and engineer new enzymes with expanded substrate scope is now proceeding faster than ever before [3]. For enzymes having two substrates, the simultaneous optimization of both substrate specificities is desired for rapidly expanding the scope of substrate acceptance, and range of products formed. Few studies have reported the engineering of both substrate specificities.

Transketolase (TK) catalyses the reversible transfer of a $C_{2}$-ketol unit from D-xylulose-5phosphate to either D-ribose-5-phosphate or D-erythrose-4-phosphate, linking glycolysis to the pentose phosphate pathway in all living cells [4, 5]. The stereospecifically controlled carbon-carbon bond forming ability of TK makes it very promising as a biocatalyst in industry, for the synthesis of complex carbohydrates and other high-value compounds [6-8]. However, to achieve industrial viability in large-scale processes, a robust transketolase toolbox is desired, in which the variants should be able to accept a diverse range of substrates including both acceptors and donors.

Wild-type TK accepts a narrow range of hydroxyaldehydes as the aldol-acceptor substrate, and with strict (2R)-specificity [9-11]. TK from E. coli has been engineered successfully for improved and reversed enantioselectivity [12], as well as an expanded aldol-acceptor substrate range including polar aliphatic [13], non-polar aliphatic [14], heteroaromatic [15, $16]$ and aromatic aldehyde derivatives $[17,18]$. Related variants were also introduced by others, into a thermostable TK from Geobacillus stearothermophilus (TKgst) [19-22].

Wild-type TKs have very limited tolerance for different donor substrates, which require both an oxo group adjacent to the $\mathrm{C}-\mathrm{C}$ bond undergoing cleavage, and a hydroxyl group at $\mathrm{C}-1$. They also show strong preference towards the trans-configuration of hydroxyls at the asymmetric C-3 and C-4 positions [11]. In addition to its natural phosphorylated donors, TK can also accept hydroxypyruvate (HPA). The use of $\beta$-hydroxypyruvate as the ketol donor 
renders the donor half-reaction irreversible, thus increasing the atom efficiency of the reaction favourably for industrial syntheses. E. coli TK accepts HPA with 30-fold higher activity than other orthologs such as from yeast and spinach, thus resulting in higher specific enzyme activity [23].

Pyruvate $\mathbf{2 d}$ differs from hydroxypyruvate only by the absence of the C-3-hydroxyl group, and yet cannot function as a substrate in the transketolase reaction, indicating the critical role of the hydroxyl group in serving as a donor substrate [24]. Unlike TK, the closely related enzyme 1-deoxy-D-xylulose-5-phosphate synthase (DXS), catalyzes the transfer of the acetyl group from pyruvate $\mathbf{2 d}$ to glyceraldehyde-3-phosphate to yield 1-deoxy-D-xylulose 5phosphate. This was used recently as a template to guide the engineering of $\mathrm{TK}_{\mathrm{gst}}$ for acceptance of pyruvate and aliphatic analogues, as the donor substrate [25]. However, this enzyme variant has not been shown to work also with the aliphatic or aromatic aldehydes as acceptor substrates. The best variants of $\mathrm{TK}_{\mathrm{gst}}$ were able to catalyse the preparative scale reactions between novel donors and hydroxylated acceptors with isolated yields of $48-$ $88 \%$. However, the acceptor substrate used during the process of directed evolution was the hydroxylated aldehyde, glycolaldehyde 1 . Therefore, as the wild-type TK already utilises hydroxylated acceptors with high activity, it remains unknown whether TK variants can be engineered to accept a combination of both pyruvate $\mathbf{2} \mathbf{d}$ as donor, and aliphatic or aromatic aldehydes as acceptors. The reactions between pyruvate $\mathbf{2} \mathbf{d}$ and aliphatic or aromatic aldehydes would allow access to a widely diverse range of products such as analogues of phenylacetylcarbinol (PAC), an important pharmaceutical intermediate, and precursors to drugs such as spisulosine and phenylpropanolamine.

In this study, we engineered $E$. coli TK variants that simultaneously accept both novel donor pyruvate $\mathbf{2} \mathbf{d}$ and unnatural aliphatic and aromatic acceptor aldehydes, including propanal 5a, pentanal $\mathbf{5 b}$, hexanal $\mathbf{5 c}$ and 3-formylbenzoic acid (3-FBA 7). Single variants capable of accepting pyruvate $\mathbf{2} \mathbf{d}$ were firstly obtained using a structure-guided strategy and previous knowledge. A small but smart library was then constructed by combining the single mutants showing improved activity towards pyruvate $\mathbf{2} \mathbf{d}$ and glycolaldehyde $\mathbf{1}$ or propionaldehyde 5a. After screening this library, we successfully obtained the TK variants that could catalyse 
the reactions between sodium pyruvate $2 d$ and propionaldehyde $5 \mathbf{a}$, or 3 -FBA 7 , with significantly improved efficiency compared to WT. The potential mechanisms for the enhanced activity was then evaluated using molecular docking simulations.

\section{Results and discussion}

\section{Design of single mutants}

Several enzymes are capable of accepting pyruvate $\mathbf{2} \mathbf{d}$ for carboligation reactions among the superfamily of ThDP-dependent enzymes [26, 27]. For example, pyruvate decarboxylase (PDC) can catalyse a carboligation side reaction that has been exploited in the asymmetric synthesis of phenylacetyl carbinol from pyruvate $\mathbf{2} \mathbf{d}$ and benzaldehyde. However, it also efficiently catalyses the decarboxylation of pyruvate $\mathbf{2} \mathbf{d}$ into acetaldehyde and $\mathrm{CO}_{2}$ showing catalytic promiscuity $[28,29]$. Therefore, we attempted to design variants of TK based on a structural alignment with PDC.

The E. coli TK crystal structure complexed with the natural donor substrate, D-xylulose-5phosphate and cofactor ThDP has been solved previously [30]. Hydroxypyruvate readily acts as a donor substrate, whereas pyruvate $\mathbf{2} \mathbf{d}$ cannot, indicating a critical role of the C-3hydroxyl group for catalysis. The TK structure in complex with the donor substrate showed that it is anchored by hydrogen bonds between the donor C-3-hydroxyl group, and His100 and His473 in the enzyme active site (Fig. 1A). Therefore, in order to stabilize new donor substrates lacking the C-3-hydroxyl group, new interactions may be required with other local residues, such as hydrophobic interactions with the methyl group in pyruvate. Therefore, we identified nine residues (His26, His66, His100, Leu116, Ile189, His261, Ser385, Asp469, His473) within $5 \AA$ of the first two carbons of the donor substrate, as potential mutation sites (Fig. 1A).

Although the TK and PDC studied only shared $6.7 \%$ sequence identity, 559 pairs of residues that are structurally equivalent were found using the TopMatch tool (Fig. S1, Table S1). The root-mean-square error of the superposition for the structurally equivalent $C_{\alpha}$ atoms was 
$2.66 \AA$ and the sequence identity in the equivalent regions increased to $12.3 \%$ (Table S1). Interestingly, only three target residues Leu116, Ser385 and His473 were located in the structurally equivalent regions (Fig. S1B). Based on structure alignment, Leu116 of TK is equivalent to lle415 of PDC, and so the mutation L116I was proposed. However, for the Ser385 and His473, the equivalent structural alignment with PDC showed the same residues at the equivalent positions (Fig. S1B). Ser385 of TK is equivalent to Ser32 of PDC, which is close to Asp28 in both the sequence and 3-D structure (Fig. 1B). Asp28 in PDC was reported previously to play a critical role in the stabilization of the enzyme-bound cofactor, and in the stereochemical control of the decarboxylation reaction [31], and so the mutation S385D was proposed. His473 formed a hydrogen bond with the C-3-hydroxy group in TK and played a role in anchoring the donor substrate. The equivalent residue in $\mathrm{TK}_{\mathrm{gst}}$ was His474, and the mutations $\mathrm{H} 474 \mathrm{~S}$ and $\mathrm{H} 474 \mathrm{~N}$ were previously found respectively to give a 6 -fold and 3-fold improved activity towards glycolaldehyde $\mathbf{1}$ and pyruvate $\mathbf{2} \mathbf{d}$. Therefore, we included the corresponding mutations $\mathrm{H} 473 \mathrm{~S}$ and $\mathrm{H} 473 \mathrm{~N}$ in E. coli TK to investigate whether these mutations would also improve the activity of E. coli TK towards pyruvate.

As for the target residues that were not in structurally equivalent regions, we proposed a range of mutations at each target site based on the types of residues found in the equivalent spatial location in PDC to enhance the chances of success. The active site residues around the cofactor and donor substrate for the aligned TK and PDC, are shown in Fig. 1B. Ile189 of TK was located in a space occupied by Glu477 in PDC, and also close to Asn488, and so the mutations I189N/E were proposed (Fig. 1B). I189Q was also included as glutamine has a similar structure to asparagine. Ile476 is a conserved hydrophobic residue among PDCs from various organisms and plays an important role in substrate recognition and enantioselectivity [32]. Three target histidines 26, 66 and 261 were all within $2 \AA$ of Ile476 in PDC, and so the mutations $\mathrm{H} 26 \mathrm{I}, \mathrm{H} 66 \mathrm{I}$ and $\mathrm{H} 261 \mathrm{l}$ were also proposed. In addition, the mutations $\mathrm{H} 26 \mathrm{Q}, \mathrm{H} 66 \mathrm{~T}$ and $\mathrm{H} 261 \mathrm{E}$ were proposed approximately corresponding to residues GIn552, Thr390 and Glu477 of PDC.

His100 in TK also formed a hydrogen bond with the C-3-hydroxyl group of the donor substrate and its mutation to alanine previously had little effect on the $K_{\mathrm{m}}$ for acceptor 
substrates, but significantly increased that of the donor substrate, suggesting its important role in accepting the donor substrate [33]. Based on the structural alignment to PDC, His100 was close to residues His114 and Phe292 of PDC (Fig. 1B), and so we proposed the mutation H100F. Considering the importance of this site, we also included alternative mutations. $\mathrm{H} 100 \mathrm{Y}$ was included as tyrosine has a similar structure to phenylalanine. In addition, the $\mathrm{TK}_{\mathrm{gst}}$ mutations $\mathrm{H} 102 \mathrm{~F} / \mathrm{L} / \mathrm{T}$ previously showed improved performance towards glycolaldehyde 1 and sodium pyruvate $\mathbf{2 d ~ [ 2 5 ] . ~ T h e r e f o r e , ~ w e ~ i n c l u d e d ~ c o r r e s p o n d i n g ~}$ mutations $\mathrm{H} 100 \mathrm{~L}$ and $\mathrm{H} 100 \mathrm{~T}$ in $E$. coli TK to investigate whether these mutations would improve activity in E. coli TK towards glycolaldehyde $\mathbf{1}$ or propionaldehyde $\mathbf{5 a}$, and pyruvate 2d.

When predicting the potential mutations, previous knowledge was also considered. Asp469 is a hotspot for engineering TK towards novel acceptor substrates. While using HPA as the donor, D469E led previously to increased activity towards aliphatic aldehyde acceptors, and D469T increased the activity towards both aliphatic and aromatic aldehydes [17, 34].

Preliminary work suggested that these two variants may have accepted pyruvate $\mathbf{2} \mathbf{d}$ as donor substrates with pentanal as the aldehyde acceptor, although with a very low reaction yield. Therefore, in addition to the $\mathrm{D} 469 \mathrm{H}$ mutation suggested by the proximity of His114 in the structurally aligned PDC, we also included D469E and D469T into our screens. In total, we designed and constructed 20 single mutants (summarised in Table S2), and measured their activities towards glycolaldehyde $\mathbf{1}$ or propionaldehyde $\mathbf{5 a}$ and pyruvate $\mathbf{2 d}$ experimentally.

\section{Characterization of single variants}

\section{Reaction with glycolaldehyde and pyruvate}

Clarified lysate preparations of the twenty single mutants were first tested for catalytic efficiency towards pyruvate $\mathbf{2 d}$, paired with glycolaldehyde $\mathbf{1}$ as this is known already to be one of the best acceptor substrates for wild-type TK. This allowed us to evaluate the acceptance of the novel donor substrate in the context of a favourable hydroxylated 
aldehyde acceptor. The target product, 3,4-dihydroxy-2-butanone (DHB) 3 of the reaction between glycolaldehyde $\mathbf{1}$ and pyruvate $\mathbf{2} \mathbf{d}$ was detected by HPLC at a retention time of $13.7 \mathrm{~min}$ (Scheme 1). However, erythrulose 4 was also detected as a side product at the retention time of $11.4 \mathrm{~min}$ (Scheme 1, Fig. 2A), which was not reported in any previous mutagenesis studies, although the reaction of two glycolaldehydes to form erythrulose is a known slow side reaction for wild-type TK [35].

Considering the possible difference in expression level of the variants, the yields of both products determined after 24-h reaction, were normalised by the TK concentration as determined from the relative density of SDS-PAGE bands, to give an approximate measure of specific activity (Fig. S2). Each of the mutations tested influenced the yields of both the target product and the erythrulose byproduct, and also the ratio of the two products, relative to WT (Fig. 2B). The variants $\mathrm{H100F/L/T/Y,} \mathrm{L116I,} \mathrm{D469E} \mathrm{and} \mathrm{H473N} \mathrm{in} \mathrm{particular,}$ gave markedly increased reaction yields of the target product relative to that of WT. All of these improved variants were subsequently purified and re-evaluated, except for H100T which was the lowest ranked. Two negative variants I189N, H473S were also purified and tested as controls (Fig. 2C). Interestingly, H100Y, L116I, D469E and H473N no longer showed an improvement in activity in their pure enzyme form, compared to wild type (WT) (Fig. 2B). The same variants also had low protein expression levels (Fig. S2) and so their poor specific activity when purified may have been due to an adverse impact of the mutations on enzyme stability, or otherwise resulted from the increased error in measuring low protein concentrations by densitometry on lysates.

The yield of reaction catalysed by H100L was $22.3 \mathrm{mM}$ (45\%) (Table 1), a 2.1-fold improvement compared to WT, consistent with previous observations with equivalent TKgst variants [25]. H100F also improved the catalytic efficiency by 1.2 -fold. However, H473N and $\mathrm{H} 473 \mathrm{~S}$ did not improve the target product yield in E. coli TK, in contrast to the effect of the equivalent mutations in TKgst (Fig. 2C). The contextual structure differences between the

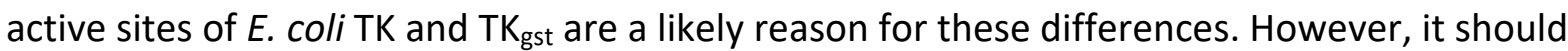
be noted that differences in the activity assays used in our work compared to that in previous reports with $\mathrm{TK}_{\mathrm{gst}}$, could also lead to similar observations. The $\mathrm{pH}$-sensitive 
colorimetric assay used for $\mathrm{TK}_{\mathrm{gst}}$ would not have detected the erythrulose byproduct, nor distinguish between the decarboxylation of pyruvate $\mathbf{2} \mathbf{d}$ versus true transketolase function (decarboxylation followed by ketone unit transfer to acceptor) $[25,36]$.

The variants also changed the relative reaction yields for the two products. WT produced equal concentrations of target product and byproduct, at $10.5 \mathrm{mM}(21 \%)$, whereas all purified variants except I189N, gave higher yields of the target product DHB 3, than the byproduct erythrulose 4 . H473N gave the highest selectivity, with no byproduct detected after 24-h reaction, even though the target product accumulated to $5.5 \mathrm{mM}$. The ratios of the target product DHB 3 relative to byproduct were $8.3,5.3,5.2,2.9,2.4$, and 1.5 , for H100L, H100F, H110Y, H473S, L116I, and D469E, respectively.

Most of the variants based on structural comparison to PDC, failed to improve the yield of the target product 3,4-dihydroxy-2-butanone $\mathbf{3}$ for the reaction between glycolaldehyde $\mathbf{1}$ and pyruvate $\mathbf{2 d}$, which is perhaps not surprising given only $6.7 \%$ sequence identity between TK and PDC, and the likely need for multiple simultaneous mutations to generate a change in function.

Reaction with propionaldehyde and pyruvate and extension to other aliphatic analogues to determine stereoselectivities

All variants were also tested for their activities towards propionaldehyde $\mathbf{5 a}$ and sodium pyruvate $\mathbf{2 d}$. Wild-type E. coli TK can accept a range of non-hydroxylated aliphatic aldehyde substrates in the presence of hydroxypyruvate. However, the activity is typically only $5-35 \%$ of those for hydroxylated substrates such as glycolaldehyde 1. Propionaldehyde $5 \mathrm{a}$ is therefore a more challenging acceptor for TK than glycolaldehyde 1, especially when using pyruvate $\mathbf{2} \mathbf{d}$ as the donor substrate. Initially the 12 variants not already purified above (Fig. 2C), were first tested for their activity in lysates, to identify any to add into the pool of purified variants. After reaction for $24 \mathrm{~h}$ using the cell lysates (Fig. 3A), the target product 3hydroxy-2-pentanone 6ad was detected indirectly using the colorimetric assay. Four variants, H26Q, H100T, H261l and D469T gave 2- to 4-fold improved specific activities 
relative to WT (Fig. 3A). These four variants were subsequently purified and re-evaluated together with the other 8 variants already purified above.

The absorbance at $450 \mathrm{~nm}$ increased with reaction time indicating a significant timedependent formation of 3-hydroxy-2-pentanone 6ad (Fig. 3B). However, the purified variants $\mathrm{H} 26 \mathrm{Q}$ and $\mathrm{H} 261$ I no longer improved the activity, even though their lysates had shown improved performance, which could also be attributed to their low expression as discussed above. Seven variants including H100F, H100L, H100T, D469E, D469T, H473N and H474S gave improved activity relative to WT, with the most active variant D469E having more than 2-fold improvement in the catalytic efficiency compared to WT (Fig. 3B).

Based on the colorimetric assay, WT also appeared to show activity towards propionaldehyde $\mathbf{5 a}$ and pyruvate $\mathbf{2} \mathbf{d}$, which had never been observed previously. In order to confirm the reaction product, GC-MS was applied to analyse the reaction mixture after 24-hours reaction. A peak was identified by GC with the retention time of $8.9 \mathrm{~min}$ (Fig. 3C). After ionization, the compound 3-hydroxy-2-pentanone 6ad revealed three expected fragments, having mass-to-charge ratios of 43,59 , and 31 , respectively, indicating the successful generation of the target product (Fig. 3D).

The peak area in GC was used to quantify the relative amounts of product generated by the enzyme reactions. We carried out the enzyme reactions for 48 hours using seven variants, including both the improved and non-improved ones in their purified form. The GC peak area and OD450 gave a good linear correlation with an $R^{2}$ of 0.93 , reflecting that the colorimetric assay was a reliable activity measurement method (Fig. S3). The negative intercept on the GC peak area axis indicated that some of the colorimetric assay response was due to background. This would reduce the apparent activity of WT considerably, although the presence of a peak by GC confirmed that it could indeed form the target product. Consistent with the measurements of the colorimetric assay, the GC peak area of the 3-hydroxy-2-pentanone 6ad showed that D469E was the most active variant, with a 4.3fold improvement in yield relative to that of WT. H100F, H473S, H100L and H473N also revealed 1.4-fold, 1.5-fold, 2.4-fold and 2.6-fold improvements in yield respectively, relative 
to WT (Fig. 3E). Larger improvements were observed by GC relative to WT, as a result of the better removal of background signal compared to in the colorimetric assay, and so the results from GC would be more accurate.

3-Hydroxy-2-pentanone 6 ad is hard to isolate due to its volatility so larger scale TK reactions were performed with pentanal $\mathbf{5 b}$ and hexanal $\mathbf{5 c}$ as acceptors with a view to isolating the products. Both pyruvate $\mathbf{2} \mathbf{d}$ and keto-butyric acid $\mathbf{2} \mathbf{e}$ were also used as donors to highlight general applicabilities of the reaction at this stage (Scheme 2). Following the screening of variants with propanal and pyruvate described above, D469E was selected for use together with D469T as a lower performing mutant for comparison purposes. As shown in Scheme 2, isolated products $6 \mathrm{bd}$ and $6 \mathrm{~cd}$ were formed in reactions with both D469T and D469E in both cases in approximately $5 \%$ yield, although some material was lost due to volatility (Table 1 ). $(3 R)$-Hydroxy-2-octanone $\mathbf{6 c d}$, the product arising from hexanal $\mathbf{5 c}$ and $\mathbf{2} \mathbf{d}$, is an insect pheromone produced by males of the longhorn beetle Anaglyptus subfasciatus and a Mosher's derivatisation method has been reported to determine enantiomeric excesses (ees) [37]. Following this precedent the TK product $6 \mathrm{~cd}$ was reacted with $(2 R)-3,3,3-$ trifluoro-2-phenylpropanoyl chloride ((R)-MTPA) to give the (2S)-Mosher's ester. ${ }^{1} \mathrm{H}$ NMR analysis revealed a major singlet for the $1-\mathrm{H}_{3}$ group at $2.21 \mathrm{ppm}$, and a minor peak at 2.13 $\mathrm{ppm}$. The positions of these two signals corresponded to the $(2 S, 3 S)$ and $(2 S, 3 R)$-esters, respectively, following the literature and indicated that the major (3S)-isomer was formed by the TK mutants: integration of the peaks revealed an ee of $>95 \%$. A second set of diastereomeric signals could also be observed for the $8-\mathrm{H}_{3}$ triplet, and integration of the signals at $0.83 \mathrm{ppm}$ (major (2S,3S)-isomer) and $0.93 \mathrm{ppm}$ (minor (2S,3R)-isomer) confirmed the ee as $>95 \%$. The absolute stereochemistry was further confirmed by measurement of the optical rotation compared to reported data (see $\mathrm{SI}$ ). In addition, racemic samples of $\mathbf{6 b d}$ and $\mathbf{6 c d}$ were also formed via chemical methods as chemical standards (see SI) to determine the isomeric compositions of the products via chiral GC which confirmed the formation of the major isomer in $>98 \%$ ee (Table 1 ). The reaction product $\mathbf{6 b d}$ formed from pentanal $\mathbf{5 b}$ and pyruvate $\mathbf{2 d}$ was then derivatised using $(R)-\mathrm{MTPA}$, and integration of the $1-\mathrm{H}_{3}$ signal again indicated an ee of $>95 \%$ for the $(S)$-isomer. Chiral GC of the racemic product compared to the TK products with D469T and D469E similarly revealed ees of $>98 \%$. These results highlighted the highly stereoselective nature of the reactions. 
From these results the same aldehydes were used with keto-butyric acid $\mathbf{2 e}$ to give $\mathbf{6 b e}$ and 6ce using D469T and D469E respectively, which gave the highest isolated yields. Racemic samples of 6 be and 6 ce were again prepared to confirm the characterisation data. To determine again the stereoselectivity for one example $\mathbf{6 b e}$, it was analysed by chiral GC and reacted again with $(R)$-MTPA. Analysis of the ester product $8-\mathrm{H}_{3}$ signal revealed two triplets at $0.82 \mathrm{ppm}$ (major $(2 S, 4 S)$-isomer) and $0.94 \mathrm{ppm}$ (minor $(2 S, 4 R)$-isomer), consistent with the method described above, with integrations indicating the ee as $>95 \%$, and GC analysis gave an ee of $>98 \%$ (see $\mathrm{SI}$ ).

The improvements in yield for the reaction between propionaldehyde $\mathbf{5 a}$ and pyruvate $\mathbf{2 d}$ from E. coli TK variants D469E, H473N, H100L, H473S and H100F, were particularly interesting given that they typically improved the activity towards glycolaldehyde 1 and sodium pyruvate $\mathbf{2} \mathbf{d}$ by a lesser extent, or even not at all for D469E, H473N and H473S. This highlights the complexity of engineering an enzyme towards two substrates simultaneously, and also that improvements towards the more hydrophobic pyruvate (relative to hydroxypyruvate), may be structurally linked to the better acceptance of the more hydrophobic aldehyde acceptor propionaldehyde 5a (relative to glycolaldehyde 1).

The catalytic reaction of TK proceeds in two stages. In the first half of the reaction, cleavage of the donor substrate is accompanied with the formation of a covalent intermediate. In the second half of the reaction, nucleophilic attack of the acceptor substrate by the enamine intermediate leads to product formation and release. The improvement of the final yield of the reaction could be attributed to both the first and second halves of the reaction. D469E showed improved reaction yields towards propionaldehyde $\mathbf{5 a}$ and pyruvate $\mathbf{2} \mathbf{d}$, and yet failed to significantly increase the activity towards glycolaldehyde $\mathbf{1}$ and pyruvate $\mathbf{2 d}$. Interestingly, D469E also showed improved activity towards propionaldehyde 5a but decreased the activity towards glycolaldehyde 1 when HPA was used as the donor $[13,16$, 36]. This suggested that D469E only improved the efficiency of the second half of the reaction which involves acceptance of the aldehyde substrate, but did not affect acceptance of pyruvate in the first half. By contrast, the mutations of His100 could have only influenced the first half of the reaction, accepting the pyruvate. We tested the correlation between 
reaction yields for the purified variants, when reacting with the two different acceptors and sodium pyruvate (Fig. S4). It clearly showed that the mutations of residue His100 were responsible for the enzyme activity towards both the glycolaldehyde and propanal, with an $\mathrm{R}^{2}$ of 0.99 when only $\mathrm{H} 100 \mathrm{~L}, \mathrm{H} 100 \mathrm{~F}, \mathrm{H} 100 \mathrm{Y}$ and $\mathrm{WT}$ were considered, suggesting that mutations of His100 were critically required to accept the pyruvate, while not being important for controlling the acceptor specificity, consistent with previous observations [33] (Fig. S4). We thus combined the variants that improved donor acceptance with those that improved acceptor acceptance, to generate a new library.

\section{Design of 'a small but smart' library}

In order to further improve the activity of $E$. coli TK towards propionaldehyde 5 a and pyruvate 2d, eight single variants including H100F, H100L, H100T, D469E, D469T, H473N, $\mathrm{H} 473 \mathrm{~S}$ and R520Q were selected for recombination to construct the library. Among them, H100F, H100L, H100T, D469E, D469T, H473N, H473S each showed improvement in the activity towards propionaldehyde $\mathbf{5 a}$ and sodium pyruvate $\mathbf{2 d}$. The R520Q mutation was also included as it was previously found to improve stability, and also the catalytic efficiency towards HPA and propionaldehyde 5a, within certain other variants [34]. This strategy kept the library size small while still sampling for potential cooperative effects between mutations. In previous work with TK [34], recombination of single active-site mutants, including at Asp469, nearly always led to the loss of soluble expression. Therefore, we used a previously stabilised variant $\mathrm{H} 192 \mathrm{P} / \mathrm{A} 282 \mathrm{P} / \mathrm{I365L} / \mathrm{G} 506 \mathrm{~A}$ (4M) as the template for constructing the new library to promote evolvability [34, 38, 39]. Introduction of these same stabilising mutations was recently found to restore the stability loss of another TK variant (S385D/D469T/R520Q) that was engineered to accepted aromatic aldehydes $[39,40]$.

\section{Improvement of activity towards propionaldehyde and pyruvate}

The 'small but smart library' was screened towards propionaldehyde $\mathbf{5 a}$ and sodium pyruvate $\mathbf{2 d}$ using the colorimetric assay. Ten promising variants were identified, purified and tested for catalytic activity. The reaction yield relative to WT was determined for the 
variants using GC after reaction for $24 \mathrm{~h}$ (Fig. 4). As the quadruple variant H192P/A282P/I365L/G506A (4M) was used as the template for constructing the library, its activity was also measured. The $4 \mathrm{M}$ maintained a comparable activity with $\mathrm{WT}$, whereas its combination with other mutations influenced their impact. The D469E variant previously gave a 4.3-fold improvement in product yield relative to the WT, whereas 4M/D469E had improved the yield by 6.7 -fold relative to $4 \mathrm{M}$, demonstrating the benefit of TK stabilisation even for the single active-site mutations (Fig. 3E \& 4). However, the addition of the compensatory stabilising $4 \mathrm{M}$ mutations did not significantly change the impact of $\mathrm{H} 100 \mathrm{~L}$ (Fig. 3E \& 4), presumably as this variant did not significantly decrease the stability of TK relative to the WT stability. Interestingly, addition of $4 \mathrm{M}$ influenced the activity of $\mathrm{H} 473 \mathrm{~N}$ in a negative way. $4 \mathrm{M} / \mathrm{H} 473 \mathrm{~N}$ only showed 1.2 -fold improvement in reaction yield compared to WT, whereas the $\mathrm{H} 473 \mathrm{~N}$ itself revealed 2.4-fold improvement. Thus, the relationship between template stability and the fitness of the new variant is not always predictable.

The combination between $\mathrm{H} 100 \mathrm{~L}$ and $\mathrm{D} 469 \mathrm{E}$ generated the variant 4M/HL/DE that gave an 8.1-fold improvement in the reaction yield compared to WT. Addition of the mutation R520Q to this variant improved the reaction yield further and generated the best variant overall, $4 \mathrm{M} / \mathrm{HL} / \mathrm{DE} / \mathrm{RQ}$, which had a 9.2-fold improvement in the reaction yield compared to WT (Fig. 4, Table 1). R520Q has been reported to increase the activity of D469T towards propionaldehyde 5a and HPA due to improved soluble protein expression. The combination of $\mathrm{H} 100 \mathrm{~L}$ and $\mathrm{H} 473 \mathrm{~N}$ also led to a variant showing 4.2-fold improvement in the reaction yield compared to the WT, confirming the important role of $\mathrm{H} 100 \mathrm{~L}$ in accepting pyruvate $\mathbf{2 d}$.

\section{Novel enzyme activity towards 3-FBA and pyruvate}

In our previous work, variants showing improved performance towards propionaldehyde 5a and HPA also improved the catalytic efficiency towards aromatic substrates with HPA, including 3-FBA 7 or 4-FBA $[17,18]$. For instance, D469T improved the specific activity towards propionaldehyde $5 \mathbf{a}$ and HPA by 5 -fold and also improved conversion yield of 3-FBA 7 and 4 -FBA from $0 \%$ to $65 \%$ and $30 \%$ respectively. We therefore explored whether any of 
the new library variants had improved activity towards the aromatic aldehyde 3-FBA 7 and pyruvate $\mathbf{2 d}$ (Scheme 3 ).

The whole library was screened for activity towards 3-FBA $\mathbf{7}$ and sodium pyruvate $\mathbf{2} \mathbf{d}$ as determined by HPLC. Most variants had no activity towards these two substrates, with the exception of 4M/H100L/D469T, 4M/D469T/H473N, and 4M/D469T/H473N/R520Q. After reaction for $24 \mathrm{~h}$, a new peak at the retention time of $4.1 \mathrm{~min}$ was identified that correlated with the consumption of the 3-FBA 7 in the HPLC chromatogram for these three variants, but not for WT or any other variants (Fig. 5A).

In order to confirm if this peak was the product expected, the peak was isolated by semipreparative HPLC and characterized by ${ }^{1} \mathrm{H}$ NMR and LC-MS. In the LC-MS analysis of the extracts, a peak with an $\mathrm{m} / \mathrm{z}$ of 195.1 was observed, corresponding to the expected product 3-(1-hydroxy-2-oxopropyl) benzoic acid 8 (Fig. S5A). The NMR spectrum also confirmed the generation of the target product (See SI). These data demonstrated that the E. coli TK variants catalysed the unprecedented reaction between the aromatic aldehyde and sodium pyruvate $\mathbf{2 d}$.

Three variants including 4M/H100L/D469T (4M/HL/DT), 4M/D469T/H473N (4M/HL/DT/HN), 4M/D469T/H473N/R520Q (4M/HL/DT/RQ) were also purified and tested for the conversion of 3-FBA 7 with sodium pyruvate $\mathbf{2 d}$ as the donor substrate. The variant 4M/H100L/D469T showed the highest activity of all, with an initial rate around 2-fold higher than those of 4M/D469T/H473N and 4M/D469T/H473N/R520Q (Fig. 5B). The variant 4M/H100L/D469T was then characterized at higher enzyme concentrations. When increasing the concentration of enzyme from $0.067 \mathrm{mg} / \mathrm{mL}$ to $1.33 \mathrm{mg} / \mathrm{mL}$, the conversion from 3-FBA 7 to product 3-(1-hydroxy-2-oxopropyl) benzoic acid 8 within $24 \mathrm{~h}$ was increased from $2.5 \%$ to $47 \%$ based on the substrate depletion (Table $1 \& \mathrm{~S} 3$ ). The specific activity, $K_{\mathrm{m}}$ and $k_{\text {cat }}$ of 4M/H100L/D469T were also found to be $0.048 \mu \mathrm{mol} \mathrm{mg}^{-1} \mathrm{~min}^{-1}, 90.4 \mathrm{mM}$ and $0.22 \mathrm{~s}^{-1}$ respectively (Table S3). 
Addition of the mutation S385Y into the double mutant D469T/R520Q previously improved the catalytic efficiency towards 3-FBA 7 and HPA by significantly decreasing the $K_{\mathrm{m}}$ [18]. We therefore introduced the S385Y mutation into the two mutants 4M/D469T/H473N and 4M/D469T/H473N/R520Q to test whether their activities could be improved. The variant 4M/H100L/D469T/H473N/R520Q was also purified for activity measurements. Interestingly, the mutation S385Y failed to improve the activity for the variants of $4 \mathrm{M} / \mathrm{D} 469 \mathrm{~T} / \mathrm{H} 473 \mathrm{~N}$ and 4M/D469T/H473N/R520Q (Fig. 5B). The residue Ser385 was within $5 \AA$ around the first two carbons of the donor substrate (Fig. 1A). The mutation S385Y, hence, could easily have interfered with the binding of pyruvate $\mathbf{2} \mathbf{d}$ and decreased the activity towards 3-FBA $\mathbf{7}$ and sodium pyruvate $\mathbf{2 d}$. The variant 4M/H100L/D469T/H473N/R520Q also showed limited activity, consistent with the activity measured using lysates (Fig. 5B).

\section{Elucidating the mechanism of enhanced activity}

To gain insights into the influence of the mutations on enzyme activity, the substrates were docked into the binding pocket of the WT and several TK variants using the Autodock Vina program [41]. 4M/H100L/D469E and 4M/H100L/D469T were selected for docking as they had significantly improved enzyme catalytic efficiencies and yet different activities towards propionaldehyde 5a and 3-FBA 7 with the only difference in their substitution of position 469. The first half of the TK reaction consists of the cleavage of the donor substrate, pyruvate $\mathbf{2} \mathbf{d}$ and the formation of the first product, carbon dioxide and a covalent intermediate, ThDP-enamine. The intermediate was first docked into wild-type and mutant TKs (Fig. S6A). ThDP-enamine had the binding affinity energy of $-9.5 \mathrm{kcal} \mathrm{mol}^{-1}$ when bound into the WT, $0.3 \mathrm{kcal} \mathrm{mol}^{-1}$ higher than when it was bound into the $4 \mathrm{M} / \mathrm{HL} / \mathrm{DE}$ and $4 \mathrm{M} / \mathrm{HL} / \mathrm{DT}$, indicating that the intermediate had stronger binding with the variants than with the WT. The pose of ThDP-enamine was similar in the binding pockets of $4 \mathrm{M} / \mathrm{HL} / \mathrm{DE}$ and $4 \mathrm{M} / \mathrm{HL} / \mathrm{DT}$ with a RMSD of $0.3 \AA$, and yet slightly to the binding in WT with a RMSD of $0.7 \AA$ (Fig. S6A, Table 2). When the pyruvate $\mathbf{2} \mathbf{d}$ instead of hydroxypyruvate was used as the donor substrate, the ( $\alpha$-hydroxyethyl)-ThDP intermediate instead of ( $\alpha, \beta$-dihydroxyethyl)ThDP intermediate was generated. In the WT, the C2 methyl group of the hydroxyethyl was located in a highly polar environment surrounded by six charged residues including $\mathrm{H} 26$, 
H66, H100, H261, D469 and H473, which was potentially unfavourable (Fig. S6B).

Substitution of histidine with leucine at the position 100 would reduce the polarity of this local region and form a hydrophobic interaction between L100 and C2 methyl group of the intermediate (Fig. S6C\&D). This could result in the slight difference of binding orientation of ThDP-enamine, and stronger binding affinity in the two variants compared to WT.

Two acceptor substrates, propionaldehyde $\mathbf{5 a}$ and 3-FBA 7 were then separately docked into the WT and variants containing the ThDP-enamine intermediate. According to the elucidated catalysis mechanisms of TK, the $\alpha$-carbanion of the ( $\alpha$-hydroxyethyl)-ThDP intermediate would attack the aldehyde group of the acceptor substrates to form the products with a carbon skeleton extended by two carbon atoms. The distances between the C-1 atom of the hydroxyethyl group of the ThDP-enamine and the carbon atom of the aldehyde group of propionaldehyde 5a were $5.2 \AA$, $4.6 \AA$ and $5.0 \AA$ for the $W T, 4 M / H L / D E$ and 4M/HL/DT respectively, consistent with the activity measurements towards propionaldehyde $\mathbf{5 a}$ and pyruvate $\mathbf{2 d}$ (Table 2 , Fig. 4 \& 6). The higher activity of $4 \mathrm{M} / \mathrm{HL} / \mathrm{DE}$ thus potentially resulted from the slightly shorter distance between the acceptor substrate propionaldehyde 5a, and the enamine intermediate, compared to $4 \mathrm{M} / \mathrm{HL} / \mathrm{DT}$ and $\mathrm{WT}$. As shown in Fig. 6 A-C, the D469T and D469E mutations apparently led to differences in the shape of the tunnel around the intermediate. The D469E mutation led to a narrower tunnel because of the longer side chain of glutamic acid. The longer side chain also has greater steric hindrance, which result in C-2, C-3 atoms of propanal have dramatically different positions when docked into WT, 4M/HL/DE and 4M/HL/DT (Fig. S7). Compared to the side chain of threonine, that of glutamic acid will have greater polarity, whereas the C-2, C-3 atoms of propanal are non-polar. The strong polarity and long side chain of Glu469, hence, may lead propanal to have a shorter distance towards the ThDP-enamine compared to WT and 4M/HL/DT (Table 2 Fig. S7).

3-FBA 7 is larger than propanal, and so the narrow substrate tunnel of $4 \mathrm{M} / \mathrm{HL} / \mathrm{DE}$ influenced the access of 3-FBA 7 to the enamine intermediate as evidenced by the distance of $6.1 \AA$, which was $1 \AA$ and $0.9 \AA$ longer than those for 3-FBA 7 docked within WT and 4M/HL/DT respectively (Table 2). This is consistent with the observation that 4M/HL/DE did not show 
any activity towards pyruvate $\mathbf{2} \mathbf{d}$ and 3-FBA 7 . By contrast, the D469T mutation expanded the size of the substrate tunnel towards the intermediate, which allowed the occurrence of nucleophilic attack of the intermediate on the aldehyde group of 3-FBA 7. This was similarly observed previously when we docked the 3-FBA 7 into D469T containing the $(\alpha, \beta$ dihydroxyethyl)-ThDP intermediate [17]. 4M/HL/DT also showed a binding affinity of -5.5 $\mathrm{kcal} \mathrm{mol}^{-1}, 0.1 \mathrm{kcal} \mathrm{mol}^{-1}$ lower than that of WT. A possible explanation is that replacing the negatively charged residue aspartic acid with the more hydrophobic and uncharged threonine provides more favourable interactions with the non-polar acceptor substrates.

Consistent with the activity test, the docking experiments showed a picture of cooperation between different residues to improve the catalytic efficiency towards different substrates. Specifically, combination between $\mathrm{H} 100 \mathrm{~L}$, responsible for the donor acceptance and D469E, responsible for propionaldehyde $\mathbf{2}$ acceptance lead to the mutation showing enhanced activity towards pyruvate and propanal, while the combination of $\mathrm{H} 100 \mathrm{~L}$ and D469T responsible for 3-FBA acceptance generate the mutation that improves the activity towards pyruvate and 3-FBA.

\section{Conclusion}

In this study, we attempted to engineer the transketolase from E. coli to accept both the unnatural acceptor substrates propanal $\mathbf{5 a}$, pentanal $\mathbf{5 b}$, hexanal $\mathbf{5 c}$ or $\mathbf{3 - F B A} \mathbf{7}$ and a novel donor substrate, sodium pyruvate $\mathbf{2} \mathbf{d}$ and its analogue keto-butyric acid $\mathbf{2 e}$, thus decreasing the polarity of both substrates. Twenty single-mutant variants were firstly designed based on structural alignment with PDC, and previous knowledge of variants in E. coli and G. stearothermophilus. The catalytic efficiency was then measured for these single-mutant variants experimentally. Beneficial mutations were then recombined using a previously obtained stable variant as the template, to construct a small but smart library. Library screening was carried out towards two reactions separately: 1) sodium pyruvate $\mathbf{2} \mathbf{d}$ and propionaldehyde $\mathbf{5 a} ; 2$ ) sodium pyruvate $\mathbf{2} \mathbf{d}$ and 3 - FBA 7 . The best variant 4M/H100L/D469E/R520Q showed a 9.2-fold improvement in the catalytic yield towards sodium pyruvate $\mathbf{2} \mathbf{d}$ and propionaldehyde $\mathbf{5 a}$ compared to WT. Pentanal and hexanal were 
also used as acceptors in the reactions with pyruvate $\mathbf{2} \mathbf{d}$ or keto-butyric acid $\mathbf{2 e}$ to determine stereoselectivities, which were found to be higher than $98 \%$ ee for the $S$ configuration. Also, three unprecedented variants were identified to be active towards the reaction between sodium pyruvate $\mathbf{2 d}$ and 3-FBA 7. The best variant 4M/H100L/D469T was able to convert $47 \%$ of substrate into product within $24 \mathrm{~h}$, whereas no conversion was observed for WT. Docking was applied to illustrate the cooperation between mutations responsible for donor acceptance and the mutation responsible for acceptor acceptance to promote the activity towards both the acceptor and donor, and this provides a guide for the future work about simultaneously engineering two substrate specificity. The hits obtained in this study have the potential to be used for developing catalytic pathways to a diverse range of products such as the phenylacetylcarbinols, an important pharmaceutical intermediate in the synthesis of drugs including spisulosine and phenylpropanolamine. This study also suggested that, in order to engineer enzymes for simultaneously accepting two novel substrates, a useful strategy is to identify the variants improving the single substrate acceptance first and then combine them together.

\section{Materials and methods}

All chemicals were obtained from Sigma-Aldrich, UK unless mentioned otherwise.

\section{Preparation of lysates for enzyme reaction}

The cell pellets from $12 \mathrm{~mL}$ cell culture were suspended using $1.2 \mathrm{~mL}$ cofactor solution containing $2.4 \mathrm{mM}$ thiamine diphosphate (ThDP), $9 \mathrm{mM} \mathrm{MgCl}$, $50 \mathrm{mM}$ Tris- $\mathrm{HCl} \mathrm{pH}$ 7.0. The suspended cells were then transferred to a $2 \mathrm{~mL}$ eppendorf tube for cell lysis by sonication. The sonicated cells were centrifuged at $13000 \mathrm{rpm}$ for $15 \mathrm{~min}$ to collect the supernatant as the cell lysates. The protein expression level was assessed by $12 \%$ sodium dodecyl sulfatepolyacrylamide gel electrophoresis (SDS-PAGE). Target bands were quantified using the GelDoc-It Imaging System (Cambridge, UK). Total protein concentrations in cell lysates were measured using the Bradford method [42]. Cell lysates were used at $2 \mathrm{mg} / \mathrm{mL}$ in enzyme reactions. 


\section{Enzyme reactions}

Enzyme reactions were carried out with lysates or purified proteins. Five acceptor substrates including glycolaldehyde $\mathbf{1}$, propanal $\mathbf{5 a}$, pentanal $\mathbf{5 b}$, hexanal $\mathbf{5 c}$ and 3 -FBA 7 were used for reaction with the donor substrates sodium pyruvate $\mathbf{2} \mathbf{d}$ or ketobutyric acid 2e. Reactions between glycolaldehyde $\mathbf{1}$ and sodium pyruvate $\mathbf{2} \mathbf{d}$ were initiated by adding $50 \mu \mathrm{L}$ or $100 \mu \mathrm{L}$ substrate solution containing $150 \mathrm{mM}$ glycolaldehyde, $150 \mathrm{mM}$ sodium pyruvate, $50 \mathrm{mM}$ Tris- $\mathrm{HCl}, \mathrm{pH}$ 7.0, into $100 \mu \mathrm{L}$ purified enzyme with the concentration of 0.2 $\mathrm{mg} / \mathrm{mL}$ or $200 \mu \mathrm{L}$ enzyme lysates with the whole protein concentration of $2 \mathrm{mg} / \mathrm{mL}$, to give a final substrate concentrations of $50 \mathrm{mM}$. Triplicate vials containing reaction solutions were incubated at $30^{\circ} \mathrm{C}$ for $24 \mathrm{~h}$. Samples were analysed by HPLC (Dionex, CA, USA) as previously [13] to determine the concentration of 3,4-dihydroxy-2-butanone 3 and erythrulose 4 against a standard curve.

Reactions between propionaldehyde $\mathbf{5 a}$ and sodium pyruvate $\mathbf{2} \mathbf{d}$ were initiated by adding $50 \mu \mathrm{L}$ or $100 \mu \mathrm{L}$ substrate solution containing $150 \mathrm{mM}$ propanal, $150 \mathrm{mM}$ sodium pyruvate 2d, $50 \mathrm{mM}$ Tris- $\mathrm{HCl}, \mathrm{pH} 7.0$, into $100 \mu \mathrm{L}$ purified enzyme with the concentration of 0.2 $\mathrm{mg} / \mathrm{mL}$ or $200 \mu \mathrm{L}$ enzyme lysates with the whole protein concentration of $2 \mathrm{mg} / \mathrm{mL}$, to give a final substrate concentrations of $50 \mathrm{mM}$. Triplicate vials containing reaction solutions were incubated at $30^{\circ} \mathrm{C}$ for $24 \mathrm{~h}$. A colorimetric assay detected the generation of the reaction product, 3-hydroxy-2-pentanone 6ad using WST-1 instead of 2,3,5-triphenyltetrazolium chloride reported previously [43]. Compared to HPA, pyruvate does not reduce the tetrazolium red, also removing the need to add carbonate resin for extracting excess HPA as previously [43]. In a 96-well microplate, $50 \mu \mathrm{L}$ reaction mixture was diluted further with 50 $\mu \mathrm{L} 50 \mathrm{mM}$ Tris- $\mathrm{HCl}$ pH 7.0, then $20 \mu \mathrm{L}$ WST-1 (0.2\%) solution and finally $10 \mu \mathrm{L} 3 \mathrm{M} \mathrm{NaOH}$ with good mixing. After incubation of $15 \mathrm{~min}$ at $25^{\circ} \mathrm{C}$, the microplate was scanned in a plate reader (BMG Fluostar Optima, Offenburg, Germany) for absorbance at $450 \mathrm{~nm}$.

Reactions between aromatic aldehyde $\mathbf{7}$ and sodium pyruvate $\mathbf{2} \mathbf{d}$ were initiated as above by adding solution containing $150 \mathrm{mM}$ 3-FBA 7, $150 \mathrm{mM}$ sodium pyruvate $\mathbf{2 d}, 50 \mathrm{mM}$ Tris- $\mathrm{HCl}$ pH 7.0 into purified enzyme solution, to give final concentration of $50 \mathrm{mM} 3-\mathrm{FBA} 7$ and 
$50 \mathrm{mM}$ sodium pyruvate $2 \mathrm{~d}$. After reaction at $30^{\circ} \mathrm{C}$ for $24 \mathrm{~h}, 20 \mu \mathrm{L}$ reaction mixture were transferred to $380 \mu \mathrm{L} 0.1 \%$ TFA to quench the reaction. Samples were then analysed by HPLC with an ACE5 C18 reverse phase column $(150 \times 4.6 \mathrm{~mm})$, UV detection at $210 \mathrm{~nm}$ and $250 \mathrm{~nm}$, and a mobile phase at a flow rate of $1.0 \mathrm{ml} / \mathrm{min}$ starting with $85 \%$ of $\mathrm{A}(0.1 \%$ TFA) and $15 \%$ of B (acetonitrile). After $0.5 \mathrm{~min}$, mobile phase B was linearly increased to $72 \%$ over 9 minutes, followed by a 2 -minute re-equilibration at $85 \%$ of $0.1 \%$ TFA and $15 \%$ acetonitrile.

The reactions between acceptor substrates pentanal $\mathbf{5 b}$ or hexanal $\mathbf{5 c}$ and the donor substrates sodium pyruvate $\mathbf{2} \mathbf{d}$ or keto-butyric acid $\mathbf{2} \mathbf{e}$ were also carried out for isolating the products and determining the stereoselectivities. The detailed procedures of TK reactions, isolating the products and measuring stereoselectivities were provided in the supporting information (see SI).

\section{Site-directed mutagenesis and library construction}

Site-directed mutagenesis was carried out with the QuikChange XL Site-Directed Mutagenesis Kit (Agilent Technologies, US), according to the manufacturer's instructions, on the TK gene in plasmid pQR791 [44], and transformed into XL10-Gold ultracompetent cells contained in the kit. Mutagenic primers were designed using the web-based QuikChange Primer Design Program (www.agilent.com/genomics/qcpd).

A small library was constructed, based on the 8 single mutants $\mathrm{H} 100 \mathrm{~F}, \mathrm{H} 100 \mathrm{~L}, \mathrm{H} 100 \mathrm{~T}$, D469E, D469T, D473N, D473S, R520Q, and combining them as described below to give 71 variants in total. Three rounds of mutagenesis were applied to construct the library. In the first round, site-directed mutagenesis was used to construct each of the following 12 variants, divided into three groups: group A were $\mathrm{H} 100 \mathrm{~F}, \mathrm{H} 100 \mathrm{~L}, \mathrm{H} 100 \mathrm{~T}$, group $\mathrm{B}$ were D469E, D469T, D473N, D473S, D469E/D473N, D469E/D473S, D469T/D473N, D469T/D473S and group C was R520Q. In the second round, pairwise recombination of the groups as $A B$, $B C$ and $A C$, was achieved by mixing templates from one group and primers from the other, to give 35 further variants. For instance, to combine group A and B mutations, the mixed PCR products of group A from the first round were used as the template, and the mixed 
mutagenic primers of group B were used as the primers for the Quikchange protocol. In the third round of $P C R$, the 24 possible new recombinants of groups $A B C$ were constructed. PCR products from each round were mixed and transformed into ultracompetent cells. The library was then screened and the hits were sequenced.

\section{Library screening}

Colonies from agar plates were picked and transferred to $2 \mathrm{~mL} 96$ deep-well square plates (DWP) filled with $900 \mu \mathrm{L} \mathrm{LB} \mathrm{Amp}{ }^{+}$medium. Three wells were inoculated with blank cells without the TK gene as the negative control. DWP plates were sealed with breathable sterile film (VMR International, US) and incubated at $37^{\circ} \mathrm{C}, 400 \mathrm{rpm}$ for $18 \mathrm{~h}$ in a humidified shaker. The final $\mathrm{OD}_{600}$ was measured from $50 \mu \mathrm{L}$ cell culture transferred into a 96-well microplate. The remaining cell culture was centrifuged at $4000 \mathrm{rpm}$ for 30 minutes to collect cell pellets, frozen at $-80^{\circ} \mathrm{C}$ and thawed at room temperature for freeze-thaw lysis of cells. The cell pellets were also suspended in $200 \mu \mathrm{L}$ lysis buffer containing $9 \mathrm{mM} \mathrm{MgCl}, 2.4 \mathrm{mM}$ ThDP, $1 \times$ bugbuster and 1\% lysonase (Merck, Hertfordshire, UK) in $50 \mathrm{mM}$ Tris- $\mathrm{HCl}$, pH 7.0 and then incubated at $25^{\circ} \mathrm{C}$ for 30 minutes. Lysates were then transferred to a normal 96well plate and centrifuged at $4000 \mathrm{rpm}$ for $30 \mathrm{~min}$, and $100 \mu \mathrm{L}$ supernatant was used in enzyme reactions in glass vials, initiated by adding $50 \mu \mathrm{L}$ aliphatic substrate solution containing $150 \mathrm{mM}$ sodium pyruvate $\mathbf{2 d}, 150 \mathrm{mM}$ propionaldehyde $5 \mathrm{a}, 50 \mathrm{mM}$ Tris- $\mathrm{HCl}, \mathrm{pH}$ 7.0 , or $50 \mu \mathrm{L}$ aromatic substrate solution containing $150 \mathrm{mM}$ sodium pyruvate $\mathbf{2 d}, 150 \mathrm{mM}$ 3- FBA 7, $50 \mathrm{mM}$ Tris- $\mathrm{HCl}, \mathrm{pH}$ 7.0. Reactions were incubated at $30^{\circ} \mathrm{C}$ for $24 \mathrm{~h}$ before detection. For reactions with sodium pyruvate $\mathbf{2} \mathbf{d}$ and propionaldehyde $\mathbf{5 a}$, the colorimetric assay described above was used to detect the product. For reactions with sodium pyruvate $2 \mathrm{~d}$ and 3-FBA 7, $20 \mu \mathrm{L}$ samples were transferred to $380 \mu \mathrm{L} 0.1 \%$ TFA to quench the reaction, centrifuged at $13000 \mathrm{rpm}$ for $15 \mathrm{~min}$, then $200 \mu \mathrm{L}$ analysed by HPLC as above.

\section{Semi-preparative HPLC}

The target peak detected by analytical HPLC was collected using semi-preparative HPLC on an Agilent ZORBAX 300SB-C18 $(250 \mathrm{~mm} \times 9.4 \mathrm{~mm}, 5 \mu \mathrm{m})$ reverse-phase column at $25^{\circ} \mathrm{C}$, 
with $2 \mathrm{~mL} / \mathrm{min}$ flow rate and detection at $250 \mathrm{~nm}$. The two column mobile phases were water with $0.1 \%$ TFA (A) and acetonitrile with $0.1 \%$ TFA (B), the column was pre-equilibrated with $85 \%$ A, 15\% B. The reaction mixture was diluted 5-fold using $0.1 \%$ TFA and then $200 \mu \mathrm{L}$ loaded onto the column. A gradient was then applied as $85 \%$ A, 15\% B at 0-5 min, then linearly ramped to $28 \%$ A, $72 \%$ B over 15 mins, and then linearly back to $85 \%$ A, $15 \%$ B over 5 mins.

\section{Gas chromatography}

After the enzyme reaction, $500 \mu \mathrm{L}$ chloroform was added and the mixture agitated, then centrifuged at $4000 \mathrm{rpm}$ for $3 \mathrm{~min}$. The organic phase was then pipetted out carefully and analysed by GC/MS with an ISQ series single Quadrupole GC-MS system (ThermoFisher Scientific, MA, US), equipped with a Rxi-5Sil MS column (30 $\mathrm{m} \times 0.2 \mathrm{~mm}$ I.D.), and the helium carrier gas at a flow rate of $1 \mathrm{~mL} / \mathrm{min}$. The column temperature was kept at $40{ }^{\circ} \mathrm{C}$ for $3 \mathrm{~min}$, then raised to $150^{\circ} \mathrm{C}$ at a rate of $2{ }^{\circ} \mathrm{C} / \mathrm{min}$, then to $200^{\circ} \mathrm{C}$ at a rate of $4{ }^{\circ} \mathrm{C} / \mathrm{min}$. The ion source temperature was $230^{\circ} \mathrm{C}$ and the range of molecular weight scanned was $20-350$ $\mathrm{m} /$ z. GC analyses were also carried out with an Agilent 7820A GC-system and a chiral stationary phase: Supelco RT BetaDEX225 column ( $30 \mathrm{~m} \times 0.25 \mathrm{~mm}, 0.25 \mu \mathrm{m}$ ) and enantiomeric excess measurements were determined with the following method: Starting temperature $100{ }^{\circ} \mathrm{C}$ and an increase of $5^{\circ} \mathrm{C}$ per minute until $135^{\circ} \mathrm{C}$, then $40{ }^{\circ} \mathrm{C}$ per minute to $210^{\circ} \mathrm{C}$.

\section{Liquid chromatography-mass spectrometry}

MS analysis was performed in the UCL Chemistry Mass Spectrometry Facility. The molecular masses of the compounds were measured on an Acquity UPLC System (Waters, MA, US) with an SQ mass spectrometer (Waters, MA, US). The HPLC was performed with a Thermo Scientific Hypersil Gold ${ }^{\mathrm{TM}} \mathrm{C} 4$ reverse phase column $(50 \times 2.1 \mathrm{~mm})$ with $0.2 \mathrm{~mL} / \mathrm{min}$ flow rate. The mobile phases were $0.1 \%(v / v)$ formic acid $(A)$ and $95 \%$ acetonitrile- $5 \%$ water $(B)$, equilibrated at $95 \% \mathrm{~A}, 5 \% \mathrm{~B}$, and then a gradient applied after sample injection to $5 \% \mathrm{~A}, 95 \%$ B over 4 mins, then back to $95 \%$ A, $5 \%$ B over 0.5 mins, and held for a further 0.5 mins. 
Compounds were ionized using electrospray ionization (ESI) and detected in positive mode. The operating condition of ESI interface were set to a capillary temperature of $300^{\circ} \mathrm{C}$, capillary voltage $9 \mathrm{~V}$ and spray voltage $4 \mathrm{kV}$.

\section{Nuclear magnetic resonance spectroscopy}

${ }^{1} \mathrm{H}$ NMR spectra were recorded on Bruker Avance 400, 500, 600 and $700 \mathrm{MHz}$ spectrometers at $25^{\circ} \mathrm{C}$, using the residual protic solvent stated as the internal standard. Chemical shifts are quoted in ppm to the nearest $0.01 \mathrm{ppm}$ using the following abbreviations: $\mathrm{s}$ (singlet), $\mathrm{d}$ (doublet), t (triplet), q (quartet), qn (quintet), sext (sextet), dd (doublet of doublets), dt (doublet of triplets), m (multiplet) defined as all multi-peak signals where overlap or complex coupling of signals make definitive descriptions of peaks difficult. The coupling constants are defined as $\mathrm{J}$ and quoted in $\mathrm{Hz} .{ }^{13} \mathrm{C}$ NMR spectra were recorded at 100,125 , 150 or $175 \mathrm{MHz}$ on Bruker Avance 400, 500, 600 and $700 \mathrm{MHz}$ spectrometers at $25^{\circ} \mathrm{C}$ using the stated solvent as standard. Chemical shifts are reported to the nearest $0.1 \mathrm{ppm}$. Melting points were measured with a Gallenkamp apparatus and were uncorrected.

\section{Structure alignment}

The TK structure 2R8O.pdb and pyruvate decarboxylase structure 2VK1.pdb were aligned using the server, TopMatch-Web $[45,46]$. The aligned structure from output was visualized using Pymol (Schrödinger, USA). Sequence identity was calculated online by the web server Sequence Identity And Similarity (SIAS, http://imed.med.ucm.es/Tools/sias.html) with the input of 2R8O.pdb and 2VK1.pdb.

\section{Computational Molecular Docking}

The structures of the variants H192P/A282P/I365L/G506A/H100L/D469E (4M/HL/DE) and H192P/A282P/I365L/G506A/H100L/D469T(4M/HL/DT) were obtained using SWISS-MODEL and 1QGD.pdb as the template [47]. ThDP-enamine, propionaldehyde 5a and 3- FBA 7 structures were drawn in Chem3D Ultra v.13.0 and energy minimised by MM2 calculations. 
Autodock Vina was used to sequentially dock the ThDP-enamine and propionaldehyde $\mathbf{5 a}$ or ThDP-enamine and 3- FBA 7 into wild-type and mutant TKs with the grid centred at -11.25 , $25.858,40.198$ and the exhaustiveness of $24[48,49]$. The grid sizes of $30 \AA \times 30 \AA \times 30 \AA, 10$ $\AA ̊$ × $10 \AA$ × $10 \AA$ and $28 \AA$ × $28 \AA$ × $28 \AA$ were used for the docking of ThDP-enamine, propionaldehyde 5a and 3- FBA 7 respectively. Five replicate dockings were carried out and the conformations with lowest energy were selected for analysis.

\section{Author Contributions}

P.A.D. designed the research. H.Y., R.I.H.L., D.S., D.M.S., S.H., and A.C.K performed the experiments. H.Y., P.A.D. and H.C.H. interpreted the data. P.A.D., H.C.H. and J.M.W. supervised the studies. H.Y., P.A.D. and H.C.H. wrote the paper. All authors reviewed, revised, and approved the manuscript for publication.

\section{Acknowledgement}

This work was supported in part by a Chinese Scholarship Council stipend (to H.Y.) and Engineering and Physical Sciences Research Council Grants EP/N025105/1 and EP/P006485/1. We gratefully acknowledge the Department of Chemistry at University College London for funding S.H. and D.S., the Biotechnology and Biosciences Research Council (BBSRC) (BB/N01877X/1) for funding D.M.S. and CONACYT is thanked for a PhD studentship to A.C.K.

\section{References}

1. Reetz, M. T., Bocola, M., Carballeira, J. D., Zha, D. \& Vogel, A. (2005) Expanding the range of substrate acceptance of enzymes: combinatorial active-site saturation test, Angew Chem Int Ed Engl. 44, 4192-6.

2. Poppe, L. \& Vertessy, B. G. (2018) The Fourth Wave of Biocatalysis Emerges- The 13 th International Symposium on Biocatalysis and Biotransformations, Chembiochem. 19, 284-287. 
3. Devine, P. N., Howard, R. M., Kumar, R., Thompson, M. P., Truppo, M. D. \& Turner, N. J. (2018) Extending the application of biocatalysis to meet the challenges of drug development, Nat Rev Chem. 2, 409-421.

4. Sprenger, G. A., Schorken, U., Sprenger, G. \& Sahm, H. (1995) Transketolase A of Escherichia coli K12. Purification and properties of the enzyme from recombinant strains, European journal of biochemistry / FEBS. 230, 525-32.

5. Draths, K. M., Pompliano, D. L., Conley, D. L., Frost, J. W., Berry, A., Disbrow, G. L., Staversky, R. J. \& Lievense, J. C. (1992) Biocatalytic Synthesis of Aromatics from D-Glucose - the Role of Transketolase, Journal of the American Chemical Society. 114, 3956-3962.

6. Demuynck, C., Bolte, J., Hecquet, L. \& Dalmas, V. (1991) Enzyme-Catalyzed Synthesis of Carbohydrates - Synthetic Potential of Transketolase, Tetrahedron Lett. 32, 5085-5088.

7. Toone, E. J. \& Whitesides, G. M. (1991) Enzymes as Catalysts in Carbohydrate Synthesis, Acs Sym Ser. 466, 1-22.

8. Morris, K. G., Smith, M. E. B., Turner, N. J., Lilly, M. D., Mitra, R. K. \& Woodley, J. M. (1996) Transketolase from Escherichia coli: A practical procedure for using the biocatalyst for asymmetric carbon-carbon bond synthesis, Tetrahedron-Asymmetr. 7, 2185-2188.

9. Turner, N. J. (2000) Applications of transketolases in organic synthesis, Curr Opin Biotechnol. 11, 527-31.

10. Kobori, Y., Myles, D. C. \& Whitesides, G. M. (1992) Substrate-Specificity and Carbohydrate Synthesis Using Transketolase, J Org Chem. 57, 5899-5907.

11. Schenk, G., Duggleby, R. G. \& Nixon, P. F. (1998) Properties and functions of the thiamin diphosphate dependent enzyme transketolase, Int J Biochem Cell Biol. 30, 1297-318.

12. Smith, M. E. B., Hibbert, E. G., Jones, A. B., Dalby, P. A. \& Hailes, H. C. (2008) Enhancing and Reversing the Stereoselectivity ofEscherichia coliTransketolaseviaSingle-Point Mutations, Advanced Synthesis \& Catalysis. 350, 2631-2638.

13. Hibbert, E. G., Senussi, T., Costelloe, S. J., Lei, W., Smith, M. E., Ward, J. M., Hailes, H. C. \& Dalby, P. A. (2007) Directed evolution of transketolase activity on non-phosphorylated substrates, J Biotechnol. 131, 425-32.

14. Hibbert, E. G., Senussi, T., Smith, M. E., Costelloe, S. J., Ward, J. M., Hailes, H. C. \& Dalby, P. A. (2008) Directed evolution of transketolase substrate specificity towards an aliphatic aldehyde, $J$ Biotechnol. 134, 240-5. 
15. Galman, J. L., Steadman, D., Bacon, S., Morris, P., Smith, M. E., Ward, J. M., Dalby, P. A. \& Hailes, H. C. (2010) alpha,alpha'-Dihydroxyketone formation using aromatic and heteroaromatic aldehydes with evolved transketolase enzymes, Chem Commun (Camb). 46, 7608-10.

16. Cazares, A., Galman, J. L., Crago, L. G., Smith, M. E., Strafford, J., Rios-Solis, L., Lye, G. J., Dalby, P. A. \& Hailes, H. C. (2010) Non-alpha-hydroxylated aldehydes with evolved transketolase enzymes, Org Biomol Chem. 8, 1301-9.

17. Payongsri, P., Steadman, D., Strafford, J., MacMurray, A., Hailes, H. C. \& Dalby, P. A. (2012) Rational substrate and enzyme engineering of transketolase for aromatics, Org Biomol Chem. 10, 9021-9.

18. Payongsri, P., Steadman, D., Hailes, H. C. \& Dalby, P. A. (2015) Second generation engineering of transketolase for polar aromatic aldehyde substrates, Enzyme and Microbial Technology. 71, 45-52.

19. Zhou, C., Saravanan, T., Lorilliere, M., Wei, D., Charmantray, F., Hecquet, L., Fessner, W. D. \& Yi, D. (2017) Second-Generation Engineering of a Thermostable Transketolase (TKGst ) for Aliphatic Aldehyde Acceptors with Either Improved or Reversed Stereoselectivity, Chembiochem. 18, 455-459.

20. Zabar, J. A., Lorilliere, M., Yi, D., Thangavelu, S., Devamani, T., Nauton, L., Charmantray, F., Helaine, V., Fessner, W. D. \& Hecquet, L. (2015) Engineering a Thermostable Transketolase for Unnatural Conversion of (2S)-Hydroxyaldehydes, Advanced Synthesis \& Catalysis. 357, 1715-1720.

21. Yi, D., Saravanan, T., Devamani, T., Charmantray, F., Hecquet, L. \& Fessner, W. D. (2015) A thermostable transketolase evolved for aliphatic aldehyde acceptors, Chem Commun (Camb). 51, 480-3.

22. Saravanan, T., Reif, M. L., Yi, D., Lorilliere, M., Charmantray, F., Hecquet, L. \& Fessner, W. D. (2017) Engineering a thermostable transketolase for arylated substrates, Green Chem. 19, 481-489.

23. Sprenger, G. A. \& Pohl, M. (1999) Synthetic potential of thiamin diphosphate-dependent enzymes, J Mol Catal B-Enzym. 6, 145-159.

24. Esakova, O. A., Meshalkina, L. E., Kochetov, G. A. \& Golbik, R. (2009) Halogenated pyruvate derivatives as substrates of transketolase from Saccharomyces cerevisiae, Biochemistry (Moscow). 74, $1234-1238$.

25. Saravanan, T., Junker, S., Kickstein, M., Hein, S., Link, M. K., Ranglack, J., Witt, S., Lorilliere, M., Hecquet, L. \& Fessner, W. D. (2017) Donor Promiscuity of a Thermostable Transketolase by Directed Evolution: Efficient Complementation of 1-Deoxy-d-xylulose-5-phosphate Synthase Activity, Angew Chem Int Ed Engl. 56, 5358-5362. 
26. Widmann, M., Radloff, R. \& Pleiss, J. (2010) The Thiamine diphosphate dependent Enzyme Engineering Database: a tool for the systematic analysis of sequence and structure relations, $B M C$ Biochem. 11, 9.

27. Hailes, H. C., Rother, D., Muller, M., Westphal, R., Ward, J. M., Pleiss, J., Vogel, C. \& Pohl, M. (2013) Engineering stereoselectivity of ThDP-dependent enzymes, FEBS J. 280, 6374-94.

28. Sandford, V., Breuer, M., Hauer, B., Rogers, P. \& Rosche, B. (2005) (R)-phenylacetylcarbinol production in aqueous/organic two-phase systems using partially purified pyruvate decarboxylase from Candida utilis, Biotechnol Bioeng. 91, 190-8.

29. Meyer, D., Walter, L., Kolter, G., Pohl, M., Muller, M. \& Tittmann, K. (2011) Conversion of pyruvate decarboxylase into an enantioselective carboligase with biosynthetic potential, J Am Chem Soc. 133, 3609-16.

30. Asztalos, P., Parthier, C., Golbik, R., Kleinschmidt, M., Hubner, G., Weiss, M. S., Friedemann, R., Wille, G. \& Tittmann, K. (2007) Strain and near attack conformers in enzymic thiamin catalysis: X-ray crystallographic snapshots of bacterial transketolase in covalent complex with donor ketoses xylulose 5-phosphate and fructose 6-phosphate, and in noncovalent complex with acceptor aldose ribose 5-phosphate, Biochemistry. 46, 12037-52.

31. Wang, J., Dong, H., Li, S. \& He, H. (2005) Theoretical study toward understanding the catalytic mechanism of pyruvate decarboxylase, J Phys Chem B. 109, 18664-72.

32. Pohl, M., Siegert, P., Mesch, K., Bruhn, H. \& Grotzinger, J. (1998) Active site mutants of pyruvate decarboxylase from Zymomonas mobilis--a site-directed mutagenesis study of L112, 1472, 1476, E473, and N482, Eur J Biochem. 257, 538-46.

33. Wikner, C., Meshalkina, L., Nilsson, U., Backstrom, S., Lindqvist, Y. \& Schneider, G. (1995) His103 in yeast transketolase is required for substrate recognition and catalysis, Eur J Biochem. 233, 750-5.

34. Strafford, J., Payongsri, P., Hibbert, E. G., Morris, P., Batth, S. S., Steadman, D., Smith, M. E., Ward, J. M., Hailes, H. C. \& Dalby, P. A. (2012) Directed evolution to re-adapt a co-evolved network within an enzyme, $J$ Biotechnol. 157, 237-45.

35. Sevostyanova, I. A., Solovjeva, O. N. \& Kochetov, G. A. (2004) A hitherto unknown transketolasecatalyzed reaction, Biochem Biophys Res Commun. 313, 771-4.

36. Yi, D., Devamani, T., Abdoul-Zabar, J., Charmantray, F., Helaine, V., Hecquet, L. \& Fessner, W. D. (2012) A pH-based high-throughput assay for transketolase: fingerprinting of substrate tolerance and quantitative kinetics, Chembiochem. 13, 2290-300. 
37. Shi, X., Leal, W. S. \& Meinwald, J. (1996) Assignment of absolute stereochemistry to an insect pheromone by chiral amplification, Bioorg Med Chem. 4, 297-303.

38. Bloom, J. D., Labthavikul, S. T., Otey, C. R. \& Arnold, F. H. (2006) Protein stability promotes evolvability, Proc Natl Acad Sci U S A. 103, 5869-74.

39. Yu, H. \& Dalby, P. A. (2018) Coupled molecular dynamics mediate long- and short-range epistasis between mutations that affect stability and aggregation kinetics, Proc Natl Acad Sci U S A. 115, E11043-E11052.

40. Yu, H. \& Dalby, P. A. (2018) Exploiting correlated molecular-dynamics networks to counteract enzyme activity-stability trade-off, Proc Natl Acad Sci U S A. 115, E12192-E12200.

41. Trott, O. \& Olson, A. J. (2010) AutoDock Vina: improving the speed and accuracy of docking with a new scoring function, efficient optimization, and multithreading, J Comput Chem. 31, 455-61.

42. Bradford, M. M. (1976) A rapid and sensitive method for the quantitation of microgram quantities of protein utilizing the principle of protein-dye binding, Analytical biochemistry. 72, 24854.

43. Smith, M. E., Kaulmann, U., Ward, J. M. \& Hailes, H. C. (2006) A colorimetric assay for screening transketolase activity, Bioorg Med Chem. 14, 7062-5.

44. Martinez-Torres, R. J., Aucamp, J. P., George, R. \& Dalby, P. A. (2007) Structural stability of E. coli transketolase to urea denaturation, Enzyme and Microbial Technology. 41, 653-662.

45. Sippl, M. J. \& Wiederstein, M. (2008) A note on difficult structure alignment problems, Bioinformatics. 24, 426-7.

46. Sippl, M. J. \& Wiederstein, M. (2012) Detection of spatial correlations in protein structures and molecular complexes, Structure. 20, 718-28.

47. Waterhouse, A., Bertoni, M., Bienert, S., Studer, G., Tauriello, G., Gumienny, R., Heer, F. T., de Beer, T. A. P., Rempfer, C., Bordoli, L., Lepore, R. \& Schwede, T. (2018) SWISS-MODEL: homology modelling of protein structures and complexes, Nucleic Acids Res. 46, W296-W303.

48. Forli, S., Huey, R., Pique, M. E., Sanner, M. F., Goodsell, D. S. \& Olson, A. J. (2016) Computational protein-ligand docking and virtual drug screening with the AutoDock suite, Nat Protoc. 11, 905-19.

49. Subrizi, F., Cárdenas-Fernández, M., Lye, G. J., Ward, J. M., Dalby, P. A., Sheppard, T. D. \& Hailes, H. C. (2016) Transketolase catalysed upgrading of l-arabinose: the one-step stereoselective synthesis of I-gluco-heptulose, Green Chem. 18, 3158-3165. 


\section{Tables}

Table 1 Reactions between novel acceptor and donor substrates catalysed by TK variants

\begin{tabular}{|c|c|c|c|c|c|c|c|}
\hline $\begin{array}{c}\text { Acceptor } \\
\text { substrates }\end{array}$ & $\begin{array}{c}\text { Donor } \\
\text { substrates }\end{array}$ & TK variant & Product & $\begin{array}{c}\text { Yield } \\
(\%)\end{array}$ & $\begin{array}{l}\text { Improvement } \\
\text { relative to WT }\end{array}$ & $\begin{array}{c}\text { Configur } \\
\text {-ation }\end{array}$ & ee $(\%)$ \\
\hline 1 & $2 d$ & $\mathrm{H} 100 \mathrm{~L}$ & 3 & $45 \%$ & 2.1-fold & ND & ND \\
\hline $5 a$ & $2 d$ & $\begin{array}{c}4 \mathrm{M}^{\mathrm{a}} / \mathrm{H} 100 \mathrm{~L} / \\
\mathrm{D} 469 \mathrm{E} / \mathrm{R} 520 \mathrm{Q}\end{array}$ & $6 a d^{b}$ & ND & 9.2 -fold & ND & ND \\
\hline $5 b$ & $2 d$ & $\mathrm{D} 469 \mathrm{E} / \mathrm{T}$ & $6 \mathrm{bd}$ & $5 \%$ & $+\infty c$ & $3 S$ & $>98 \%^{\mathrm{d}}$ \\
\hline $5 b$ & $2 e$ & D469T & 6be & $4 \%$ & $+\infty c$ & $4 S$ & $>98 \%^{d}$ \\
\hline $5 c$ & $2 d$ & $\mathrm{D} 469 \mathrm{E} / \mathrm{T}$ & $6 \mathrm{~cd}$ & $5 \%$ & $+\infty c$ & 35 & $>98 \%^{\mathrm{d}}$ \\
\hline $5 c$ & $2 e$ & D469E & $6 c e$ & $7 \%$ & $+\infty c$ & ND & ND \\
\hline 7 & $2 d$ & $\begin{array}{c}\text { 4M/H100L/ } \\
\text { D469T }\end{array}$ & 8 & $47 \%$ & $+\infty c$ & ND & ND \\
\hline
\end{tabular}

a4M contains mutations H192P/A282P/I365L/G506A.

${ }^{b}$ Difficult to isolate due to its high volatility.

'No reaction was observed for WT.

${ }^{\mathrm{d} D e t e r m i n e d ~ b y ~ G C . ~}$

Table 2 Substrates binding pose and affinity in wild-type and mutant TKs

\begin{tabular}{|c|c|c|c|c|c|c|c|c|c|c|c|}
\hline & \multicolumn{3}{|c|}{ ThDP-enamine } & \multicolumn{4}{|c|}{ Propionaldehyde } & \multicolumn{4}{|c|}{$3-F B A$} \\
\hline & $\begin{array}{c}\text { Affinity } \\
(\mathrm{kcal} \\
\left.\mathrm{mol}^{-1}\right) \\
\end{array}$ & RM & $D^{[a]}$ & $\begin{array}{c}\text { Affinity } \\
(\mathrm{kcal} \\
\left.\mathrm{mol}^{-1}\right)\end{array}$ & $\begin{array}{c}\text { Distance } \\
{ }^{\mathrm{a}}(\AA)\end{array}$ & & & $\begin{array}{c}\text { Affinity } \\
(\text { kcal } \\
\left.\text { mol }^{-1}\right)\end{array}$ & $\begin{array}{c}\text { Distance } \\
{ }^{\mathrm{a}}(\AA)\end{array}$ & $\mathrm{RN}$ & \\
\hline WT & -9.5 & 0 & 0.7 & -2.4 & 5.2 & 0 & 2.0 & -5.4 & 5.0 & 0 & 1.1 \\
\hline 4M/HL/DE & -9.8 & 0.7 & 0 & -2.4 & 4.6 & 2.0 & 0 & -5.6 & 6.1 & 1.1 & 0 \\
\hline $4 \mathrm{M} / \mathrm{HL} / \mathrm{DT}$ & -9.8 & 0.7 & 0.3 & -2.4 & 5.0 & 0.9 & 2.4 & -5.5 & 5.1 & 2.1 & 2.5 \\
\hline
\end{tabular}

${ }^{a}$ The RMSD was calculated with WT or $4 M / H L / D E$ as the reference

${ }^{b}$ The distance is the nucleophilic attack distance of $\alpha$-carbanion of the ThDP-enamine on the aldehyde group of the acceptor substrate 


\section{Schemes}<smiles>O=CCO</smiles>

1
$+$

TK<smiles>CC(=O)[O-]</smiles>

2d

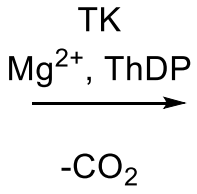

$\mathrm{CO}_{2}$<smiles>CC(=O)C(O)CO</smiles>

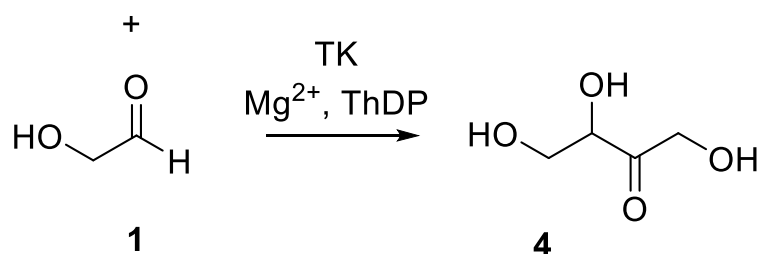

Scheme 1 TK mediated the reaction between glycolaldehyde 1 and pyruvate $2 \mathrm{~d}$. Target product 3,4-dihydroxy-2-butanone (DHB) 3 and side product erythrulose 4 were generated. 


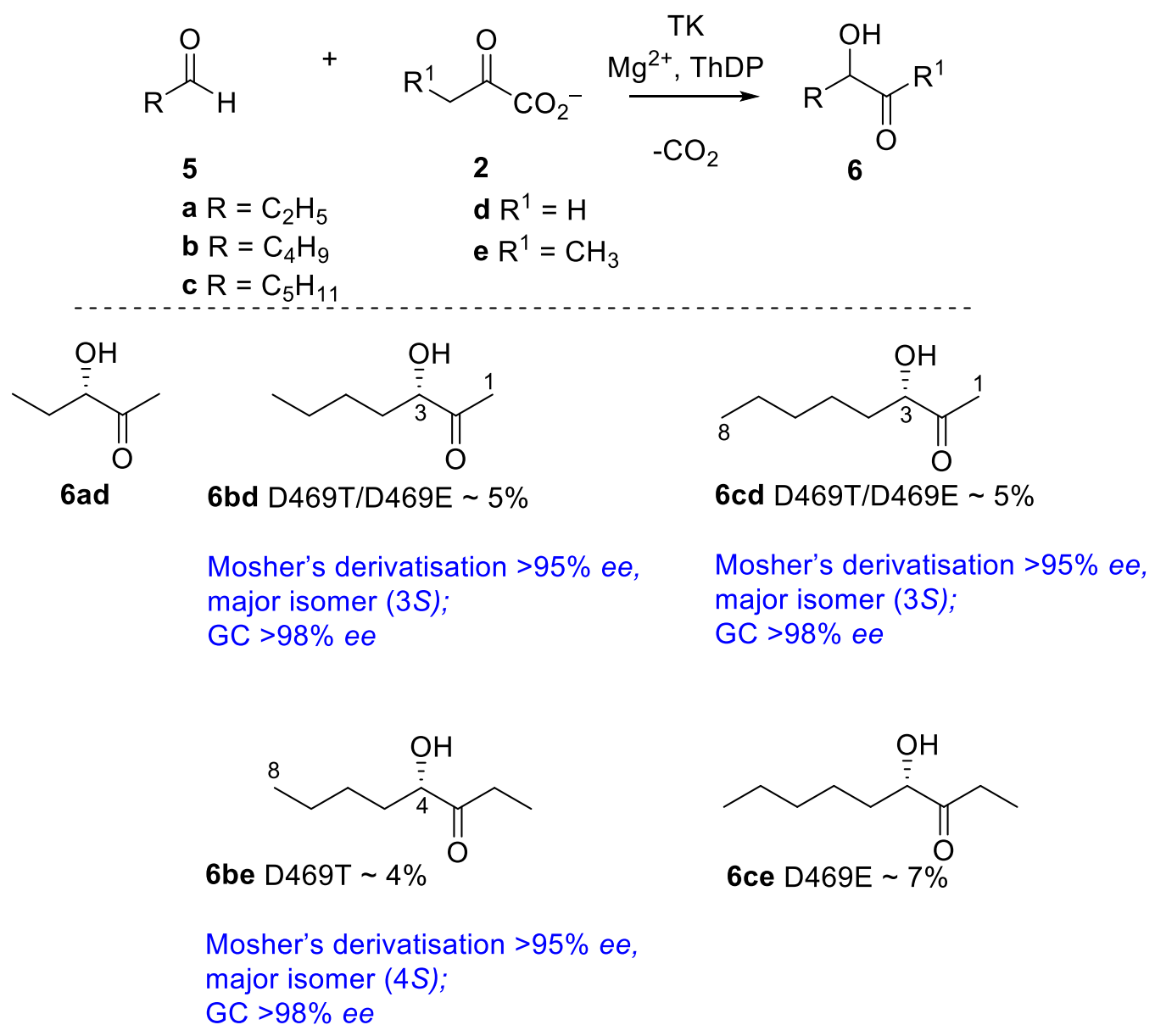

Scheme $\mathbf{2}$ TK mediated the reactions between aliphatic acceptor aldehydes and donor substrates including pyruvate $\mathbf{2 d}$ and keto-butyric acid $2 \mathrm{e}$.<smiles>O=Cc1cccc(C(=O)O)c1</smiles>

7<smiles>CC(=O)O[Na]</smiles>

2d

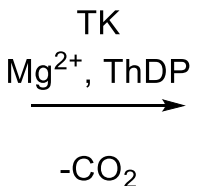<smiles>CC(=O)C(=O)c1cccc(C(=O)O)c1</smiles>

8

Scheme 3 TK mediated the reaction between aromatic aldehyde 3-formylbenzoic acid 7 and pyruvate $\mathbf{2 d}$. 


\section{Figures}

A

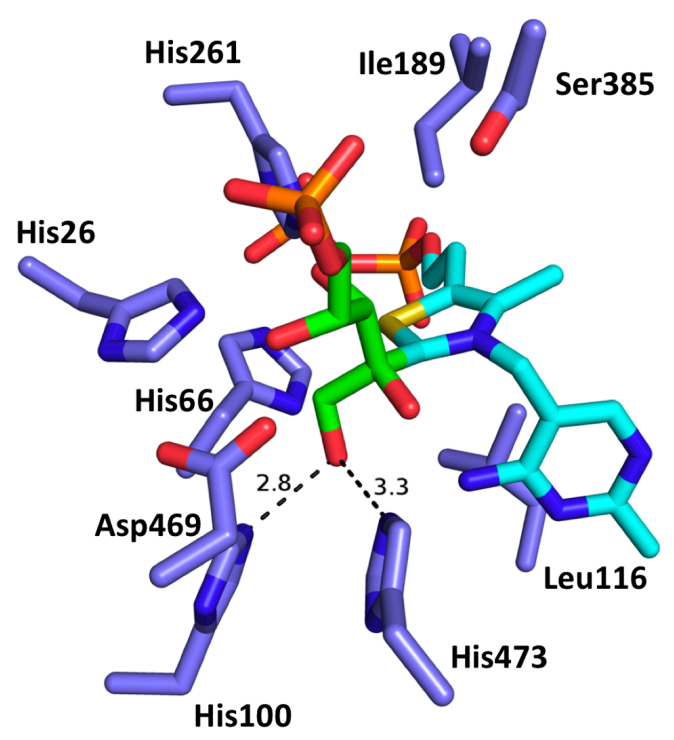

B

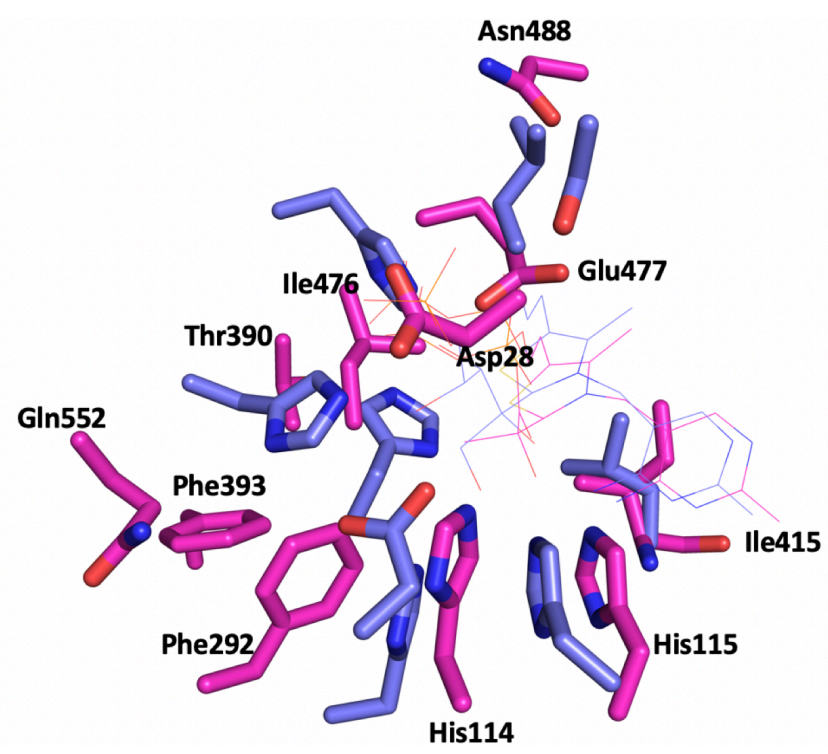

Fig. 1. Structure alignment between transketolase (2R8O.pdb) and pyruvate decarboxylase (2VK1.pdb). A, Structure of TK in the complex with D-xylulose-5-phosphate: green, the substrate; cyan, thiamine pyrophosphate (ThDP). Distances are shown in $\AA$. B, Active-site alignment between transketolase (blue) and pyruvate decarboxylase (pink). Only the residues of pyruvate decarboxylase are labelled. The cofactor ThDP of two enzymes are shown as lines. 

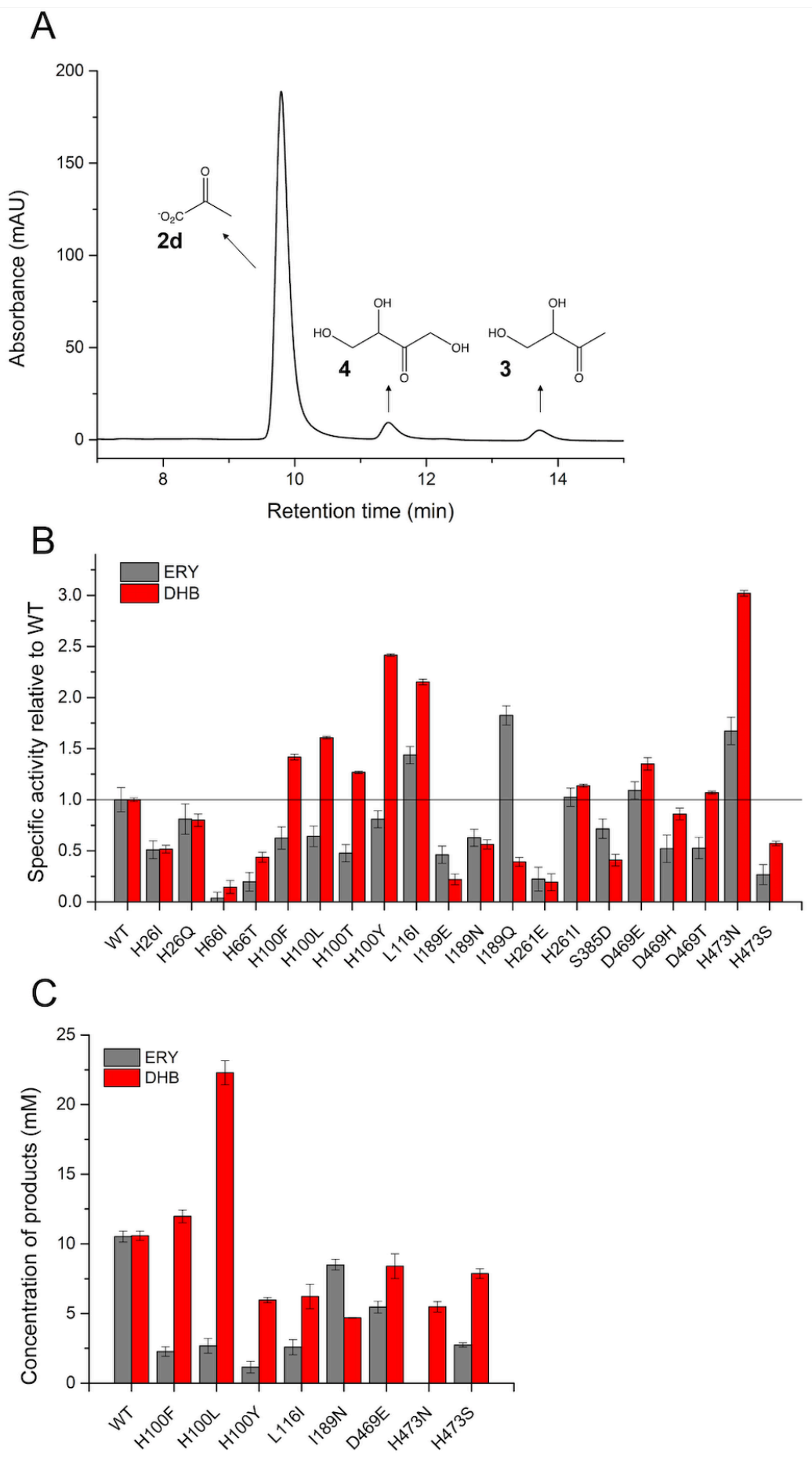

Fig. 2. Mutants catalysing the reaction between glycolaldehyde $\mathbf{1}$ and sodium pyruvate $\mathbf{2 d}$. A, HPLC chromatography of the reaction glycolaldehyde $\mathbf{1}$ and sodium pyruvate $\mathbf{2 d}$. Retention time for erythrulose (ERY) 4 and 3,4-dihydroxy-2-butanone (DHB) 3 were $11.4 \mathrm{~min}$ and $13.7 \mathrm{~min}$, respectively. $\mathrm{B}$, Specific activity (yield at $24 \mathrm{~h}$ normalised to TK concentration) of TK variant lysates, relative to WT, for reaction of glycolaldehyde $\mathbf{1}$ and sodium pyruvate $\mathbf{2 d}$. C, Final concentrations of the two products for the 24-h reactions catalysed by the purified variants. Enzyme reactions were carried out at $50 \mathrm{mM}$ glycolaldehyde $1,50 \mathrm{mM}$ sodium pyruvate $2 \mathrm{~d}, 50 \mathrm{mM}$ Tris $-\mathrm{HCl}, \mathrm{pH} 7.0$, and $0.13 \mathrm{mg} / \mathrm{mL}$ purified enzyme or enzyme from $1.3 \mathrm{mg} / \mathrm{mL}$ lysate, in triplicate vials, at $30^{\circ} \mathrm{C}$ for $24 \mathrm{~h}$. 
A

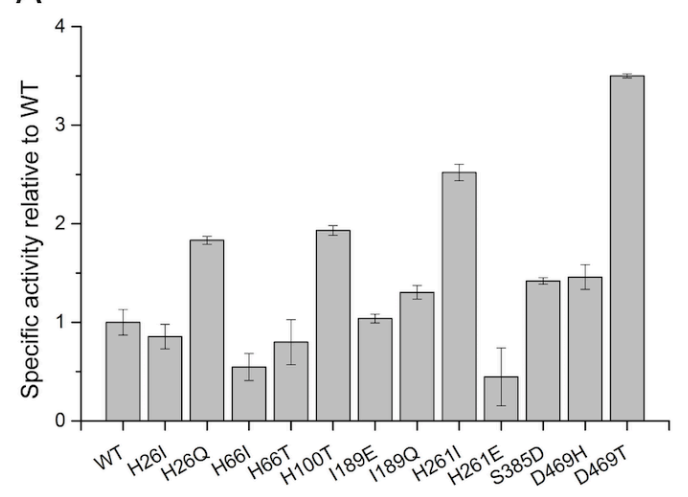

C

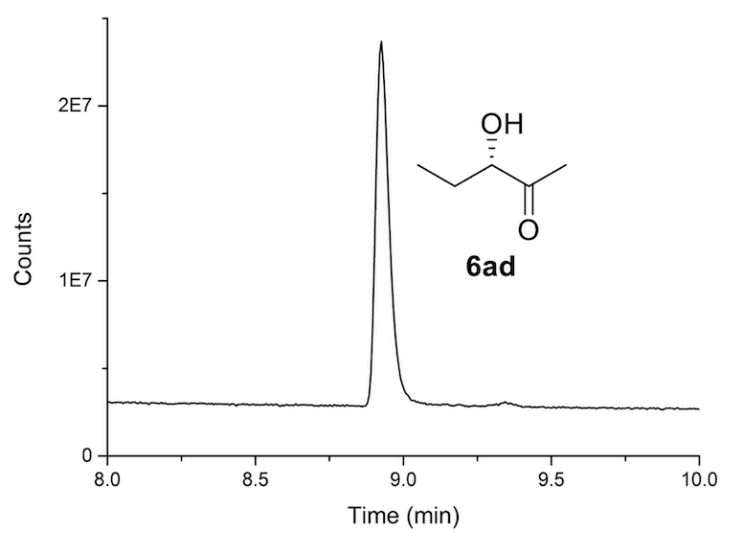

B

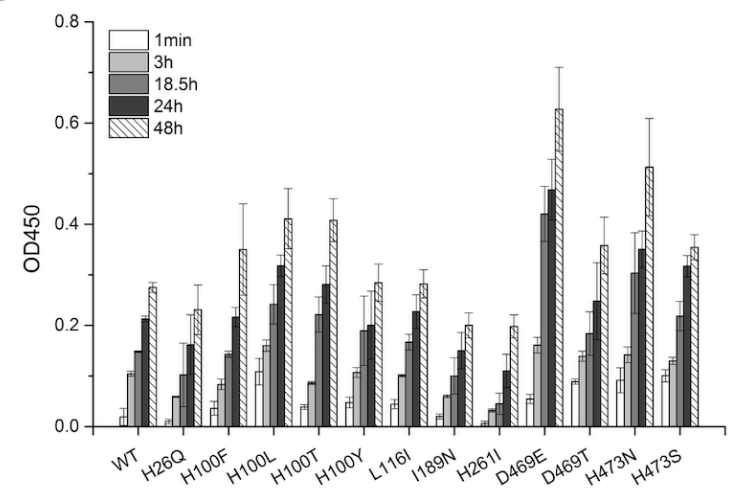

E

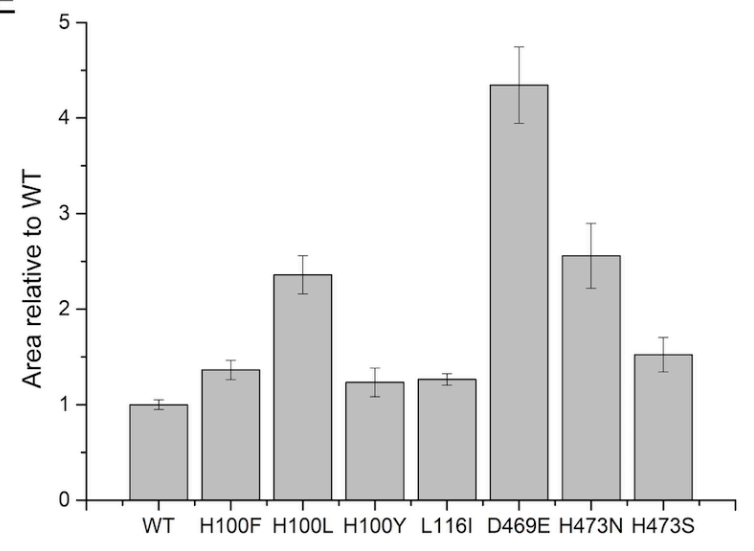

D

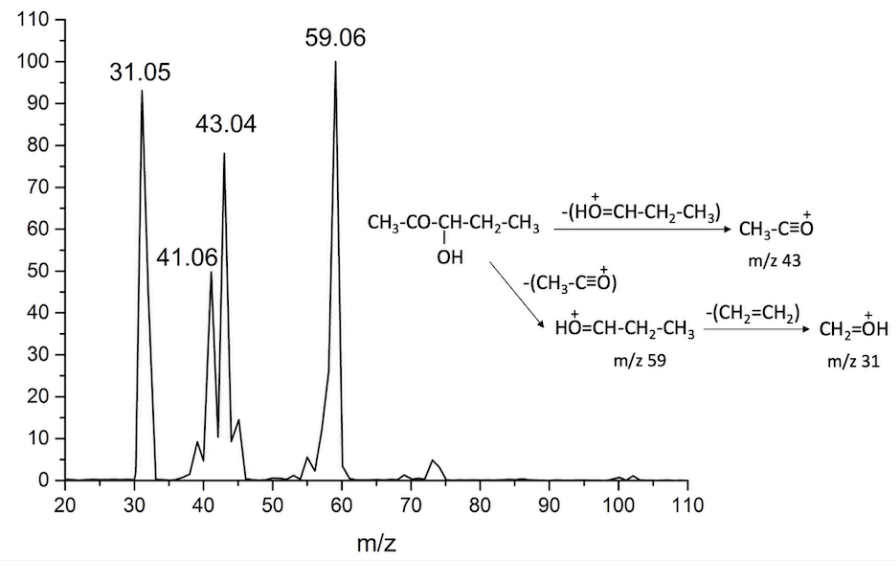

Fig. 3. Activity of TK variants towards propionaldehyde $\mathbf{5 a}$ and sodium pyruvate $\mathbf{2 d}$. (A), Specific activity (yield at $24 \mathrm{~h}$ normalised to TK concentration) of TK variant lysates, relative to WT, for reaction of propionaldehyde $\mathbf{5 a}$ and sodium pyruvate $\mathbf{2 d}$. (B), the yield of enzyme reactions catalysed by purified proteins based on the colorimetric assay. (C), the peak of 3-hydroxy-2pentanone 6ad identified by GC for WT. (D), mass spectrum of the 3-hydroxy-2-pentanone 6ad GC peak fraction. (E), yield of 48-h reaction quantified based on GC peak area. Enzyme reactions were carried out at $50 \mathrm{mM}$ propionaldehyde $5 \mathrm{a}, 50 \mathrm{mM}$ sodium pyruvate $\mathbf{2 d}, 50 \mathrm{mM}$ Tris- $\mathrm{HCl}, \mathrm{pH}$ 7.0, and $0.13 \mathrm{mg} / \mathrm{mL}$ purified enzyme or enzyme from $1.3 \mathrm{mg} / \mathrm{mL}$ lysate, in triplicate vials, at $30{ }^{\circ} \mathrm{C}$ for $24 \mathrm{~h}$ or $48 \mathrm{~h}$. 


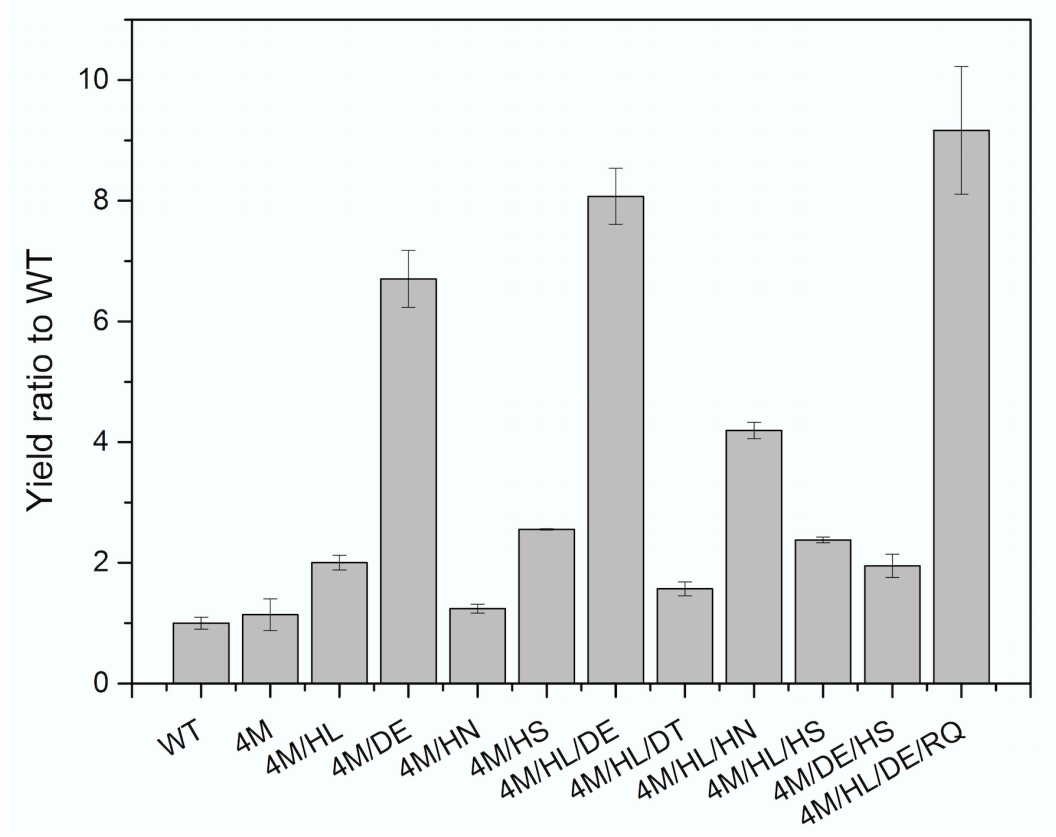

Fig. 4. Yield of reactions towards propionaldehyde and pyruvate, catalysed by purified TK variants for $24 \mathrm{~h}$. The yield was calculated based on GC area. $4 \mathrm{M}$ is the quadruple mutant H192P/A282P/I365L/G506A. HL, H100L; DE, D469E; HN, H473N; HS, H473S; RQ, R520Q. The catalytic activity was tested towards $50 \mathrm{mM}$ propionaldehyde $5 \mathbf{a}$ and $50 \mathrm{mM}$ sodium pyruvate $2 \mathbf{d}$ in $50 \mathrm{mM}$ Tris- $\mathrm{HCl}$ solution at $30^{\circ} \mathrm{C}$. The enzyme concentration used was $0.13 \mathrm{mg} / \mathrm{mL}$. 

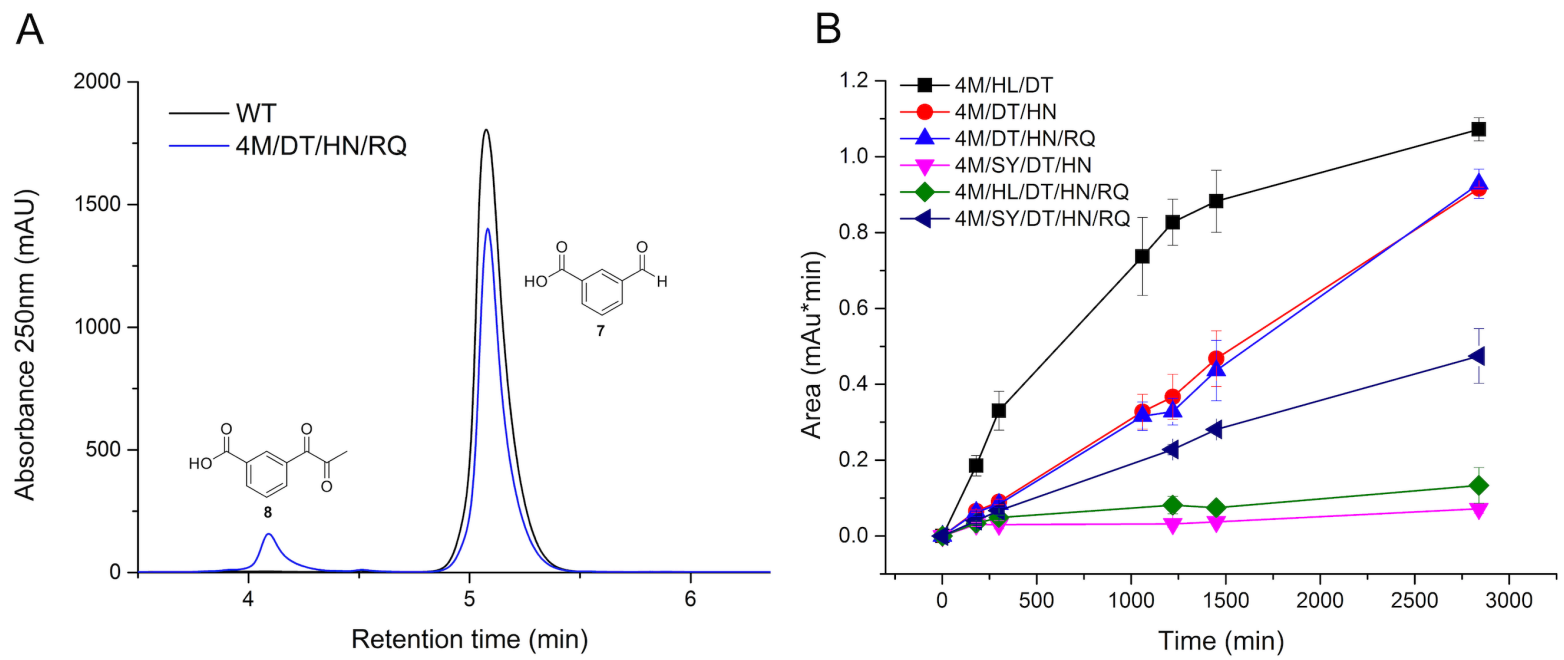

Fig. 5. Catalytic reaction between $3-$ FBA 7 and sodium pyruvate $\mathbf{2 d}$. A, HPLC chromatograms of reaction between 3- FBA 7 and sodium pyruvate $2 \mathrm{~d}$. B, Catalytic activity of TK variants. $4 \mathrm{M}$ is the quadruple mutant H192P/A282P/I365L/G506A. HL, H100L; SY, S385Y; HN, H473N; DT, D469T; RQ, R520Q. The enzyme activity was tested towards $50 \mathrm{mM} 3-\mathrm{FBA} 7$ and $50 \mathrm{mM}$ sodium pyruvate $\mathbf{2 d}$ in $50 \mathrm{mM}$ Tris- $\mathrm{HCl}$ solution at $30^{\circ} \mathrm{C}$. And the enzyme concentration used was $0.067 \mathrm{mg} / \mathrm{mL}$. 

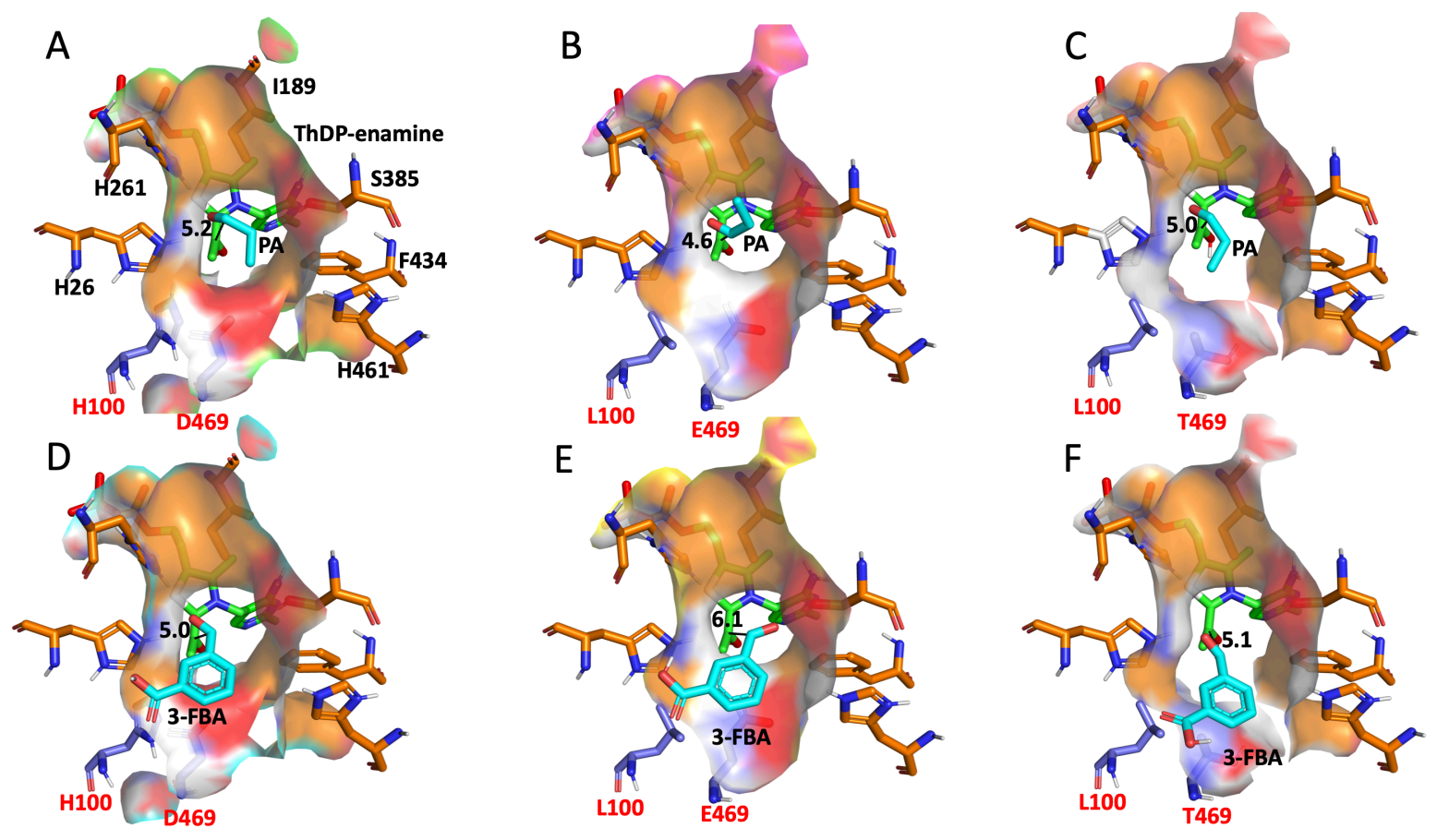

Fig. 6. Binding mode analysis of substrates in wild-type and mutant TKs. A, Docking of propionaldehyde $\mathbf{5 a}$ into WT containing the ThDP-enamine intermediate; $\mathrm{B}$, Docking of propionaldehyde $5 \mathrm{a}$ into $4 \mathrm{M} / \mathrm{HL} / \mathrm{DE}$ containing the ThDP-enamine intermediate; C, Docking of propionaldehyde 5 a into $4 \mathrm{M} / \mathrm{HL} / \mathrm{DT}$ containing the ThDP-enamine intermediate; $\mathrm{D}$, Docking of 3FBA 7 into WT containing the ThDP-enamine intermediate; E, Docking of 3- FBA 7 into 4M/HL/DE containing the ThDP-enamine intermediate; F, Docking of 3-FBA 7 into $4 \mathrm{M} / \mathrm{HL} / \mathrm{DT}$ containing the ThDP-enamine intermediate. Lightblue, mutational residues; Orange, active site residues which were only labelled in WT; Green, ThDP-enamine intermediate; Cyan, acceptor substrates including Propionaldehyde 5a and 3- FBA 7. The unit of the distance was angstrom. Residues surrounding the substrate-binding pocket are shown in surface representation. 


\section{Supporting information}

Additional supporting information may be found online in the supporting information section at the end of the article.

Table S1 TK (2R8O.pdb) and PDC (2VK1.pdb) structure alignment parameters

Table S2 Design of single variants

Table S3 Characteristics of the variant 4M/HL/DT

Fig. S1 Structure alignment between transketolase (2R8O.pdb) and pyruvate decarboxylase (2VK1.pdb)

Fig. S2 Densitometry to measure TK variants expression in the lysates with the protein concentration of $2 \mathrm{mg} / \mathrm{mL}$

Fig. S3 Correlation between colorimetric assay and the GC-MS measurement for the reactions yield

Fig. S4 Activity correlation between reactions with sodium pyruvate as donor and with glycolaldehyde or propionaldehyde as acceptor

Fig. S5 LC-MS analysis of the reaction product between 3-FBA and sodium pyruvate

Fig. S6 Pose of ThDP-enamine intermediate in the binding pockets of wild-type and mutant TKs

Fig. S7 Structural alignment of TK wild type and two variants 4M/HL/DE, 4M/HL/DT

Appendix S1 General experimental for synthesis and analysis

Appendix S2 General procedures for TK reactions

Appendix S3 Stereoselectivity measurements

Appendix S4 NMR spectra 